\title{
The Kinematic Structure of Merger Remnants
}

\section{Citation}

Cox, T. J., Suvendra N. Dutta, Tiziana Di Matteo, Lars Hernquist, Philip F. Hopkins, Brant Robertson, and Volker Springel. 2006. "The Kinematic Structure of Merger Remnants." The Astrophysical Journal 650 (2): 791-811. https://doi.org/10.1086/507474.

\section{Permanent link}

http://nrs.harvard.edu/urn-3:HUL.InstRepos:41381796

\section{Terms of Use}

This article was downloaded from Harvard University's DASH repository, and is made available under the terms and conditions applicable to Other Posted Material, as set forth at http:// nrs.harvard.edu/urn-3:HUL.InstRepos:dash.current.terms-of-use\#LAA

\section{Share Your Story}

The Harvard community has made this article openly available.

Please share how this access benefits you. Submit a story.

\section{Accessibility}


Draft Version OCtober 3, 2018

Preprint typeset using $\mathrm{LAT}_{\mathrm{E} X} \mathrm{X}$ style emulateapj v. 6/22/04

\title{
THE KINEMATIC STRUCTURE OF MERGER REMNANTS
}

\author{
T. J. Cox ${ }^{1}$, Suvendra N. Dutta ${ }^{1}$, Tiziana Di Matteo ${ }^{2}$, Lars Hernquist ${ }^{1}$, Philip F. Hopkins ${ }^{1}$, Brant Robertson ${ }^{1}$, \\ AND VOLKER SPRINGEL ${ }^{3}$ \\ Draft version October 3, 2018
}

\begin{abstract}
We use numerical simulations to study the kinematic structure of remnants formed from mergers of equal-mass disk galaxies. In particular, we show that remnants of dissipational mergers, which include the radiative cooling of gas, star formation, feedback from supernovae, and the growth of supermassive black holes, are smaller, rounder, have, on average, a larger central velocity dispersion, and show significant rotation compared to remnants of dissipationless mergers. The increased rotation speed of dissipational remnants owes its origin to star formation that occurs in the central regions during the galaxy merger. We have further quantified the anisotropy, three-dimensional shape, minor axis rotation, and isophotal shape of each merger remnant, finding that dissipational remnants are more isotropic, closer to oblate, have the majority of their rotation along their major axis, and are more disky than dissipationless remnants. Individual remnants display a wide variety of kinematic properties. A large fraction of the dissipational remnants are oblate isotropic rotators. Many dissipational, and all of the dissipationless, are slowly rotating and anisotropic. The remnants of gas-rich major mergers can well-reproduce the observed distribution of projected ellipticities, rotation parameter $(\mathrm{V} / \sigma)^{*}$, kinematic misalignments, $\Psi$, and isophotal shapes. The dissipationless remnants are a poor match to this data. We also investigate the properties of merger remnants as a function of initial disk gas fraction, orbital angular momentum, and the mass of the progenitor galaxies. Our results support the merger hypothesis for the origin of low-luminosity elliptical galaxies provided that the progenitor disks are sufficiently gas-rich, however our remnants are a poor match to the bright ellipticals that are slowly rotating and uniformly boxy.
\end{abstract}

Subject headings: galaxies: ellipticals — evolution — formation — interactions — methods:N-body simulations

\section{INTRODUCTION}

The observed absorption-line spectra and red colors of elliptical galaxies suggest that their stars were formed at high redshift $(z \geq 1)$ and that very little star formation has occurred in them since then. According to the "merger hypothesis" (Toomre \& Toomre 1972; Toomre 1977), these red elliptical galaxies are produced by the collision and merger of spiral galaxies, and hence the progenitors of present day ellipticals may be high-redshift spirals. While relatively little is known about disk galaxies at high redshift, it is likely that these disks were more concentrated and gas-rich than their low-redshift counterparts. Indeed, preliminary observational evidence (Erb et al. 2006) indicates that galaxies at redshift $z \approx 2$ do have large gas fractions $f_{\text {gas }} \sim 0.5$, with some approaching $f_{\text {gas }} \sim 0.8-0.9$. Thus, any attempt to understand the formation, properties and scaling relations of the present day population of elliptical galaxies, within the context of the "merger hypothesis", must consider gas-rich mergers.

Requiring that the disk galaxy progenitors contain a significant fraction of gas is nothing new to the "merger hypothesis". One of the main objections to this mechanism of producing ellipticals, argued by, for instance, Ostriker (1980), is that the centers of ellipticals are more

\footnotetext{
1 Harvard-Smithsonian Center for Astrophysics, 60 Garden Street, Cambridge, MA 02138, USA

2 Carnegie-Mellon University, Dept. of Physics, 5000 Forbes Ave., Pittsburgh, PA 15213, USA

3 Max-Planck-Institut für Astrophysik, Karl-Schwarzchild-
} Straße 1, 85740 Garching bei München, Germany concentrated than local spirals. Cast in terms of phasespace density, this objection states that the high central phase-space density of ellipticals cannot be produced by the merger of low phase-space spirals because, according to Liouville's Theorem, phase-space density is conserved during a collisionless process (Carlberg 1986). However, this argument breaks down when the merger constituents contain gas, which can radiate energy, and hence increase the phase space density (Lake 1989). An estimate of how much gas is required to match the central densities of ellipticals was provided by Hernquist et al. (1993), who used N-body simulations and analytic arguments to suggest that mergers of spiral galaxies containing $\geq 30 \%$ gas would be sufficient to account for the high phase space densities of ellipticals.

Within the context of the hierarchical theory of structure formation, gas-rich major mergers may play a much larger role than just resolving the central phasespace densities of elliptical galaxies. Previously, we have described a "cosmic cycle" of galaxy formation and evolution in which gas-rich mergers drive the evolution of quasars (Hopkins et al. 2006a), induce the growth of supermassive black holes (Di Matteo et al. 2005), and produce red elliptical galaxies (Springel et al. 2005a; Hopkins et al. 2006c) that obey many of the observed scaling relations (Robertson et al. 2006a b). A schematic view of this picture is presented in Fig. 1 of Hopkins et al. (2006a). However, the success of this scenario also must be gauged by its ability to produce remnants that have kinematic and morphological properties characteristic of observed ellipticals. It is this question 
that we address in the current paper.

Observations indicate that galaxy spheroids can be classified into two groups (Davies et al. 1983; Bender et al. 1989; Bender 1988; Faber et al. 1997; Kormendv \& Bender 1996, and references therein). Large, luminous spheroids have hot gaseous halos, boxshaped isophotes, surface-brightness profiles with flat "cores," show very little rotation and are almost uniformly classified as ellipticals. Less luminous spheroids tend to have little, if any, hot gas, disk-shaped isophotes, power-law surface-brightness profiles, and exhibit rotation that is along the photometric major axis. The latter group encompasses many low-luminosity ellipticals, bulges and S0s.

The last of these properties, the alignment and rotation of spheroidal galaxies, may be the result of a more fundamental dichotomy among elliptical galaxies; that is, the isotropy of their velocity distributions. Because spheroids can be flattened for (at least) two reasons, rotation and velocity anisotropy, the lack of rotation in large ellipticals suggests that these systems have significant velocity anisotropy, while many low-luminosity ellipticals and bulges are consistent with being isotropic systems flattened by their observed rotation.

One viewpoint is that these two classes of elliptical galaxies exhibit different properties because they are formed via different mechanisms. Along this line of reasoning, Naab \& Burkert (2003) argue that large ellipticals are formed by the dissipationless merger of two comparable-mass disk galaxies, i.e., major mergers, while low-luminosity ellipticals are produced by the dissipationless merger of unequal mass disks, i.e., minor mergers. To demonstrate this possibility, Naab \& Burkert (2003) used numerical simulations of dissipationless disk galaxy mergers to show that remnants of major mergers rotate very little and are, in general, boxy, similar to luminous ellipticals. On the other hand, the simulated minor mergers rotate significantly and have disky isophotes, similar to low-luminosity ellipticals. However, these simulations did not include gas physics, and hence would not induce starbursts, quasar activity, nor satisfy the scaling relations of elliptical galaxies (Robertson et al. 2006a). Moreover, if most ellipticals are relatively old, i.e. have mainly old stellar populations, and were formed long ago by mergers, it is likely that the progenitor galaxies would have been gas-rich and had a higher gas fraction than local large, star-forming galaxies.

In this paper we use a large suite of numerical simulations to explore the kinematic properties of remnants produced by the merger of comparable-mass disk galaxies. We specifically address the differences between remnants formed via dissipationless mergers versus those produced in gas-rich mergers that include the cooling of gas, star formation and feedback. We find that gasrich mergers can successfully reproduce many of the kinematic properties of observed elliptical galaxies, while dissipationless remnants provide a poor match the data.

The organization of the rest of this paper is as follows. In $\S 2$ we describe the numerical simulations, including the disk galaxy models and the galaxy collisions (2.1), followed by the techniques employed to analyze individual merger remnants (2.2). In $\S 3$ we present the results of our analysis for the entire series of merger remnants. To begin, we report the aggregate of measured properties for our entire series of simulated merger remnants (3.1). Following this, we show the remnant rotational support (3.2), including where this rotation originates (3.3), and what individual remnants are like (3.4). To better characterize the remnants we also analyze their anisotropy (3.5), shape (3.6), minor-axis kinematics (3.7), and how these quantities depend on our various input assumptions (3.9). Finally, in $\S 4$ we discuss the implications of our results for the formation of elliptical galaxies and we conclude in $\S 5$

\section{METHODS}

\subsection{Merger Simulations}

The simulations presented here are part of a large, ongoing effort to study galaxy mergers and how this process impacts the formation and evolution of galaxies. Hence, the methods used here are identical to, and also described in, several related works (Cox et al. 2006; Di Matteo et al. 2005; Hopkins et al. 2005a, 2006a, 2005b c, 2006b c, 2005d; Lidz et al.|2006; Robertson et al. 2006a, 2005, 2006b; Springel et al. $2005 \mathrm{a}$ b). Readers that desire a more detailed description of the simulation code and the construction of the progenitor galaxy models are referred to Springel et al. $(2005 \mathrm{~b})$, where the majority of the methods were first introduced. Below, we provide a brief overview of our methodology and focus on the assumptions most relevant for the results reported here.

The simulations described here were performed using the N-body/SPH (smoothed particle hydrodynamics) code GADGET2 (Springel 2005), which is based on a fully conservative formulation of SPH (Springel \& Hernquist 2002). Along with the standard features of this publically available code, the version we employ includes radiative cooling of gas, star formation that is designed to match the observed Schmidt-law (Schmidt 1959; Kennicutt 1998), the multiphase feedback model of Springel \& Hernquist (2003) softened $\left(q_{\mathrm{EOS}}=0.25\right)$ so that the mass-weighted interstellar medium temperature is $\sim 10^{4.5} \mathrm{~K}$, and a centrally located sink particle that can accrete gas and release isotropic thermal energy that represents a massive black hole (Springel et al. 2005b).

The fiducial galaxy model consists of a dark matter halo and an embedded rotationally-supported exponential disk. For simplicity we do not include a spheroidal bulge in our fiducial galaxy model. The dark matter halo is initialized with a Hernquist (1990) profile that has an effective concentration of 9 , a spin parameter $\lambda=0.033$, and a circular velocity $V_{200}=160 \mathrm{~km} \mathrm{~s}^{-1}$. The exponential disk size is fixed by requiring that the disk mass fraction ( $4.1 \%$ of the total mass) is equal to the disk spin fraction. This results in a radial disk scale length of $3.9 \mathrm{kpc}$. In addition, a specified fraction $f$ of the disk mass is in a gaseous component. The galaxy models are realized by 120,000 dark matter particles, and 80,000 disk particles. A fraction $f \times 80,000$ of the disk particles represent the gaseous disk, and the remainder represent the collisionless stellar disk.

In the present study, we restrict our analysis to simulations of major mergers between identical disk galaxies. We also consider only two types of progenitor disks. One, where the exponential disk is purely stellar $(f=0)$, and a second where the disk is $40 \%$ gas $(f=0.4)$. In all simulations, we adopt a gravitational softening length 
TABLE 1

Disk Orientations

\begin{tabular}{lccccl}
\hline \hline $\begin{array}{c}\text { Run } \\
(1)\end{array}$ & $\begin{array}{c}\theta_{1} \\
(2)\end{array}$ & $\begin{array}{c}\phi_{1} \\
(3)\end{array}$ & $\begin{array}{c}\theta_{2} \\
(4)\end{array}$ & $\begin{array}{c}\phi_{2} \\
(5)\end{array}$ & \multicolumn{1}{c}{$\begin{array}{c}\text { Comments } \\
(6)\end{array}$} \\
\hline $\mathrm{h}$ & 0 & 0 & 0 & 0 & both prograde \\
$\mathrm{b}$ & 180 & 0 & 0 & 0 & prograde-retrograde \\
$\mathrm{c}$ & 180 & 0 & 180 & 0 & both retrograde \\
$\mathrm{d}$ & 90 & 0 & 0 & 0 & polar 1 \\
$\mathrm{e}$ & 30 & 60 & -30 & 45 & tilted 1 \\
$\mathrm{f}$ & 60 & 60 & 150 & 0 & polar 2 \\
$\mathrm{g}$ & 150 & 0 & -30 & 45 & tilted 2 \\
$\mathrm{i}$ & 0 & 0 & 71 & 30 & Barnes orientations \\
$\mathrm{j}$ & -109 & 90 & 71 & 90 & $\Downarrow$ \\
$\mathrm{k}$ & -109 & -30 & 71 & -30 & \\
$\mathrm{l}$ & -109 & 30 & 180 & 0 & \\
$\mathrm{~m}$ & 0 & 0 & 71 & 90 & \\
$\mathrm{n}$ & -109 & -30 & 71 & 30 & \\
$\mathrm{o}$ & -109 & 30 & 71 & -30 & \\
$\mathrm{p}$ & -109 & 90 & 180 & 0 & \\
& & & & &
\end{tabular}

Note. - List of disk galaxy orientations for major merger simulations. Col. (1) is our unique orientation identification. Cols. (2) and (3) are the initial orientation of disk 1 and cols. (4) and (5) are the orientation of disk 2. A brief description of several unique orientations is listed in column (6).

of $140 \mathrm{pc}$. We restrict our analysis to quantities measured on the scales of the effective radius, i.e., several kpc, where we are confident that we have sufficient resolution. Unfortunately, with this resolution we cannot reliably determine the inner $(\leq 140 \mathrm{pc})$ surface density profiles (Lauer et al. 2005, and references therein) nor follow the merger of the binary black holes (see, e.g., Milosavliević \& Merritt 2001).

Once built, pairs of identical galaxies are placed on parabolic orbits with the spin axis of each disk specified by the angles $\theta$, and $\phi$ in standard spherical coordinates. Table 1 lists the adopted orientation of each progenitor for our fifteen simulated mergers. The orientation list contains seven idealized mergers (labeled $b-h$ ) that represent orientations often seen in the literature. For example, orientation $h$ has the galactic spin and orbital angular momentum aligned in what is typically called a prograde-prograde merger. Also included are slight variations of this case, e.g., prograde-retrograde, retrograderetrograde, and polar. The remainder of the mergers (labeled $i-o$ ) follow Barnes (1992) in that they are selected to be unbiased initial disk orientations according to the coordinates of two oppositely directed tetrahedra. These orbits are identical to those explored by e.g. Naab \& Burkert (2003) and therefore allow for a direct comparison to their results.

The primary results of our work are based upon two series of simulated major mergers. The first series consists of the fifteen orientations listed in Table 1 each simulated with progenitor disks composed of purely collisionless stellar mass $(f=0)$. The second series is identical to this first series but the progenitor disks all contain $40 \%$ gas $(f=0.4)$. In what follows we will consistently refer to the former series as "dissipationless" mergers, while the latter are "dissipational", or " $40 \%$ gas" mergers.

In $\S 3.9$ we consider additional simulations to address how robust our results are to the chosen initial conditions.

\subsection{Remnant Analysis}

The techniques we employ to analyze the merger remnants are designed to mirror those typically adopted by observers. For each remnant we project the stars onto a plane as if observed from a particular direction. An example of the projected stellar mass is shown in the upper-left plot of Figure 1 The stellar mass is composed of two components. One, the dissipationless disk particles that are present in our initial conditions, and two, stars that are formed during the simulation from dense gas, when included.

Once the stellar mass is projected onto a plane, we determine the iso-density contour that encloses half of the stellar mass and fit an ellipse to it. We label the semimajor axis of this ellipse $a$, and the semi-minor axis $b$. The ellipticity is then defined as $\epsilon=1-b / a$. We note that $a$ is slightly larger than the half-mass radius $R_{e}$, which is typically calculated using a circular aperture. Both the circular-aperture half-mass radius, and the fitted halfmass ellipse are overlaid on the projected surface density in Figure 11 In addition, the iso-density contours are shown. The remnant shown in Figure 1 is a dissipational merger from the $f$ orbit. It is a fast rotator viewed from an angle perpendicular to the orbital plane.

We note that observations often determine $R_{e}$ in a manner which differs from our approach. In that case, the surface brightness profile is fit to an analytic function, e.g., a $R^{1 / 4}$ (de Vaucouleurs 1948) or Sersic (Sersic 1968) profile, from which the effective radius is extracted. Unfortunately, owing to the limited resolution of telescopes and finite backgrounds, this technique is subject to uncertainties that depend on the radial range over which the fit is performed and the assumed profile (Truiillo et al. 2001). In fact, Bovlan-Kolchin et al. (2005) show that the $R_{e}$ of Hernquist (1990) profile can be underestimated by nearly $30 \%$ when the maximum radius used for the fitting produces is varied between $\sim 2 R_{e}$ to $>10 R_{e}$. Because of these complications, and because we know the exact location of all stellar material we can extract the half-mass radius directly from the particle information. This eliminates any ambiguity between the procedure used to fit the profile and the resultant $R_{e}$. In what follows we will typically use $a$, rather than $R_{e}$, as a measure of the "size" of our merger remnants.

To quantify the kinematics of each remnant, we place a slit along the major axis and measure the velocity and dispersion along the slit. Again, this is demonstrated in Figure 1 where we show the projected two dimensional rotation and dispersion velocity fields, the slit, and the resultant velocity profiles. The slit has length and width of $\sim 3 a$, and $0.25 a$, respectively. The slit is divided into 26 bins, lengthwise, and the line of sight velocity field is measured for each bin. For simplicity, the velocity profile in each bin is assumed to be Gaussian, and thus we extract the mean rotation velocity and velocity dispersion from each bin.

The rotation velocity $V_{\text {maj }}$ along the major axis is determined as the average absolute value of the maximum and minimum velocity along the slit. The velocity dispersion $\sigma$ of each remnant is the average of all dispersions $R<0.5 a$ along the slit. In practice, the dispersion profile is fairly flat out to $\sim R_{e}$ and thus the choice of 0.5 does not affect our results. The large aperture used to 


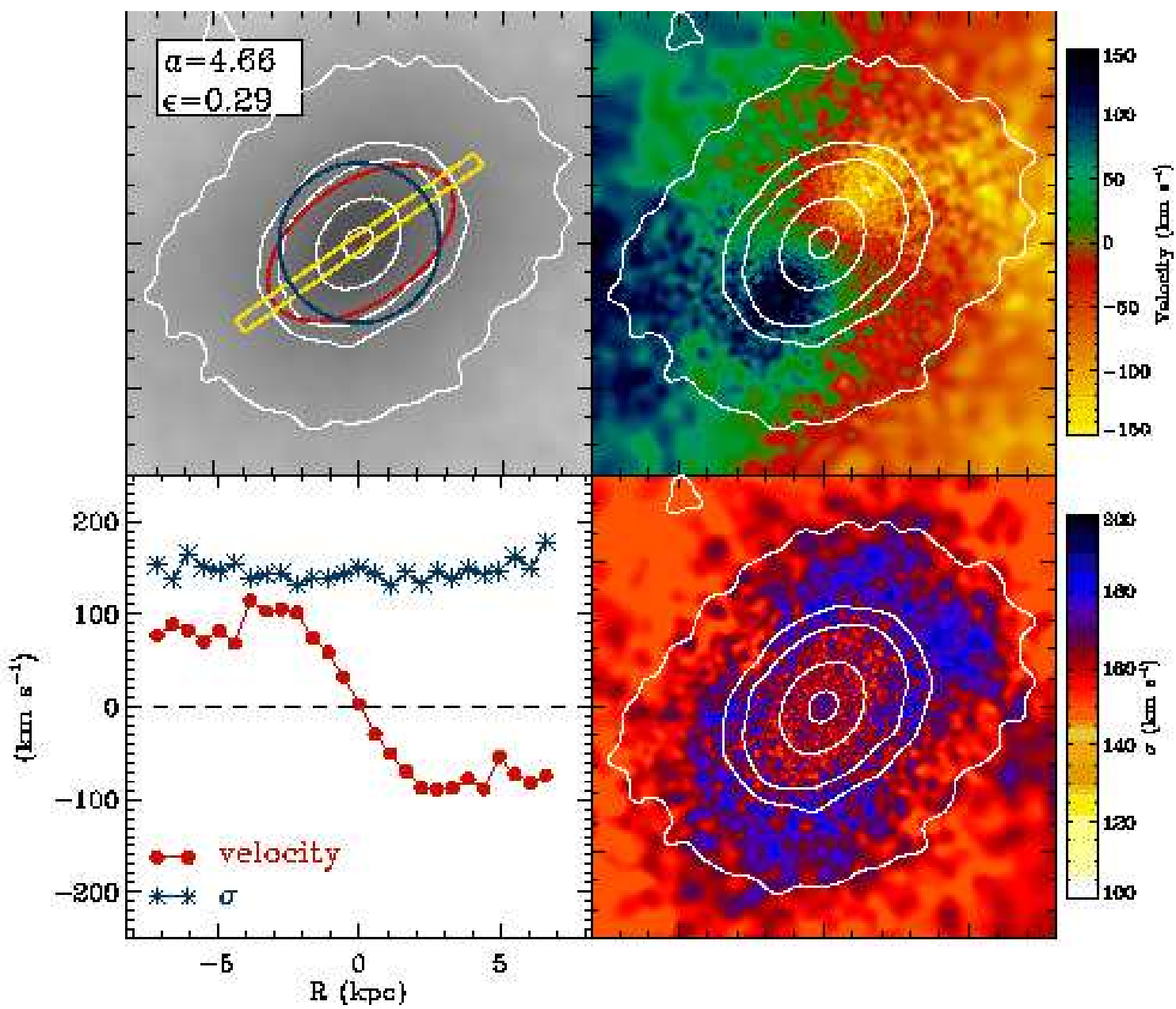

FIG. 1. - Projected mass density (upper left) and velocity (upper right) and dispersion (lower right) fields for one of our merger remnants (dissipational merger, orientation $f$, viewed perpendicular to the orbital plane) with overlaid isodensity contours. The half-mass radius, computed within a circular aperture, is shown by a (blue) circle. The half-mass isodensity contour has been fit with a (red) ellipse. The semi-major axis length $a$ and ellipticity $\epsilon$ are shown in the upper-left of the figure. A slit has been placed along the major axis and the velocity along the slit is show in the lower left panel. This remnant is a fast rotator.

measure $\sigma$ is also selected becuase it is much greater than the numerical resolution.

We note that the two dimensional velocity fields contain a wealth of information that is largely lost by the use of a simple slit. However, the majority of velocity data taken to date has been obtained using slits and we therefore follow this procedure in our work here. We do note, though, that a fruitful avenue for future work is the comparison of simulated two dimensional velocity fields such as those presented in Figure1 to observational samples such as SAURON (Cappellari et al. 2006), which use integral field spectroscopy and thus image the twodimensional velocity field.

Because our models do not account for cosmological evolution, the simulation time is not directly connected to redshift. The initial conditions are designed to approximate our own Milky Way galaxy except, when included, a higher disk gas fraction. Thus, the mergers presented here are probably more representative of moderate redshifts where disks were likely gas-rich. In fact, if the Milky Way has been forming stars at a steady rate of $1 \mathrm{M}_{\odot} \mathrm{yr}^{-1}$, without any other mass flux, it would have had a gas fraction above $40 \%$ about 10 Gyr ago $(z \sim 1.5)$. In any case, we will mainly compare our remnants to local elliptical galaxies, most of which are old stellar systems and dynamically relaxed. Hence, we would like to ensure that the merger remnants are relaxed systems as well. In order to investigate the dynamical stability of our remnants, Figure 2 shows the size, both $a$ and $R_{e}$, ellipticity $\epsilon$, major axis rotation speed $V_{\text {maj }}$, central velocity dispersion $\sigma$, and the ratio $V_{\mathrm{maj}} / \sigma$ as a function of time after the merger is complete for the same remnant that was shown in Figure 1 For the most part, the remnant relaxes quickly $(<300 \mathrm{Myr})$ after the merger. Prior to this, the central dispersion $\sigma$, the rotation speed, and size appear to be the most strongly time-dependent quantities. In particular, the dispersion is about $\sim 15 \%$ larger immediately after the merger compared to the relaxed remnant. Of all the quantities, the rotation speed fluctuates the most, and it varies within $10 \%$ of its average value, throughout the simulation. These $10 \%$ fluctuations are carried over to the ratio $V_{\text {maj }} / \sigma$, and thus we consider this a reasonable quantification of the error associated with when we observe each remnant, which we take to be $\sim 2.5$ Gyr after the merger is complete.

As a final comment, we note that all results presented 


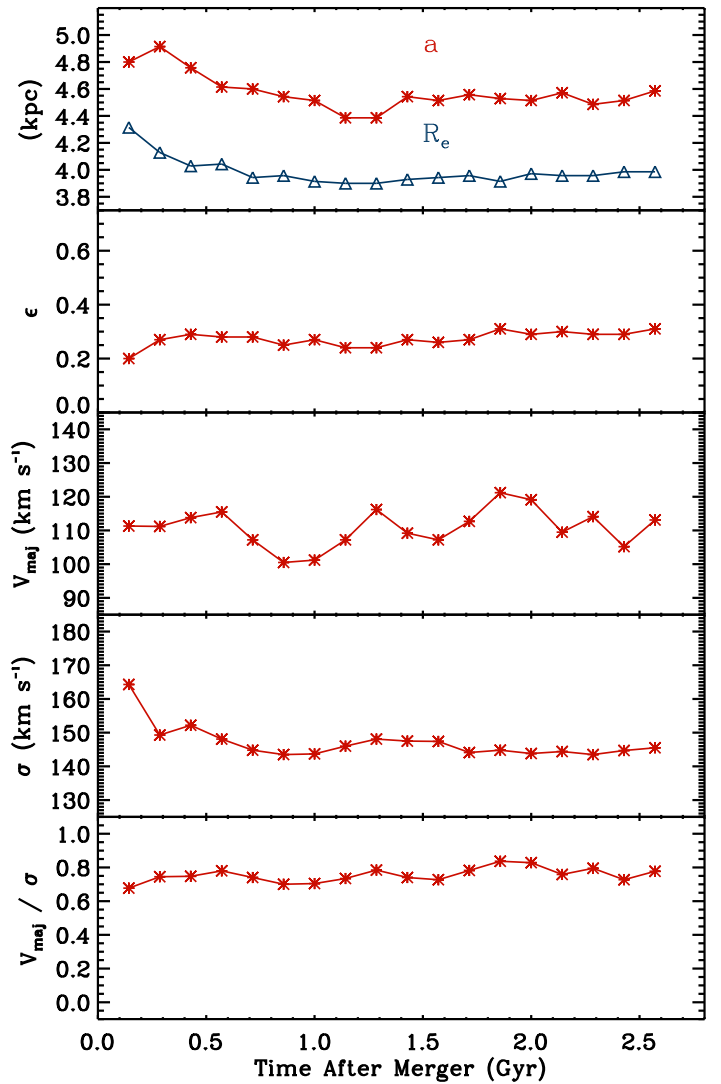

FIG. 2.- Time evolution of the semi-major axis $a$, half-mass radius $R_{e}$, ellipticity $\epsilon$, major axis rotation speed $V_{\text {maj }}$, central velocity dispersion $\sigma$, and the ratio $V_{\text {maj }} / \sigma$ for the same merger remnant displayed in Figure 1

in this work use the stellar mass as opposed to the stellar light. This choice is motivated in part by the uncertain star formation history of all particles designated as stars at the beginning of the simulation. As we show in $\S 3.3$ the stars formed during the simulation tend to rotate more than those present at the beginning of the simulation, and thus our choice to weight the velocity by mass as opposed to luminosity may underestimate the velocities as compared to recent merger remnants while being a fair representation of most old ellipticals.

\section{RESULTS}

\subsection{Effective Radius, Ellipticity, Dispersion, and Rotation}

Figure 3 shows histograms of the semi-major axis $a$, half-mass isophote ellipticity $\epsilon$, central velocity dispersion $\sigma$, and maximum rotation speed along the major axis $V_{\text {maj. }}$ Each of the 30 remnants (15 dissipational, and 15 dissipationless) is projected along 195 lines of sight. The angles are selected using the HEALPIX (Górski et al. 2005) software with nside $=4$ and uniformly sample a unit sphere. This procedure results in a total of 2250 "data" points which are displayed in Figure 3 for both the dissipationless and the dissipational merger remnants, represented by the (blue) open and (red) cross-hatched histograms, respectively.

It is apparent that dissipational merger remnants are quite different from their dissipationless counterparts.
The effects of gaseous dissipation and star formation result in remnants that are more compact, rounder, have higher velocity dispersion, and have a much more uniform distribution of rotation speeds.

The size of the remnants is a strong function of dissipation. In fact, every dissipational remnant is smaller (by nearly $\sim 50 \%$ ) than its corresponding dissipationless version. This fact is unsurprising given the significant gas fraction of our progenitor disks and the efficiency with which mergers drive gas into the galaxy centers (Barnes \& Hernquist 1991, 1996), where it fuels a burst of star formation (Mihos \& Hernquist 1996). For the mergers we present here, this process is so efficient that the average half-mass radius $R_{e}$ of the dissipational remnants, $4.0 \mathrm{kpc}$, is smaller than the original progenitor disk $4.4 \mathrm{kpc}$. The average $R_{e}$ of the dissipationless remnants is $6.9 \mathrm{kpc}$. The average semi-major axis $a$ of the dissipational remnants is $4.1 \mathrm{kpc}$ and $7.9 \mathrm{kpc}$ for the dissipationless remnants.

To check the absolute size of our merger remnants, we note that the size-mass relationship found in the Sloan Digital Sky Survey (SDSS) for early-type galaxies suggests a typical size of $3.4 \mathrm{kpc}$ for galaxies of equivalent stellar mass $\left(\sim 8 \times 10^{10} \mathrm{M}_{\odot}\right)$ to our simulated remnants (Shen et al. 2003). Hence, it appears that both our dissipational and dissipationless remnants are larger than SDSS galaxies (see also Robertson et al.|2006a). However, as pointed out by Bovlan-Kolchin et al. (2005, Appendix A), sizes can be systematically underestimated depending on the range of radii considered when fitting an assumed profile. We also note our initial disk galaxies are relatively large, a reflection of an above average spin parameter $\lambda$ assumed for the dark matter halo. In reality there exists a distribution of spins and thus disk sizes. Furthermore, Robertson et al. (2006a) have shown that the sizes of the remnants depend somewhat on the orbit of the merger, in agreement with Bovlan-Kolchin et al. (2005), and matching the observations would thus require a distribution of orbits consistent with those expected cosmologically. Therefore, we consider this (dis)agreement as marginal at best and here we simply note that the dissipational remnants are in closer agreement with the SDSS size-mass relation for early-type galaxies than dissipationless remnants.

The top-right plot in Figure 3 shows the distribution of ellipticities for our merger remnants. Overplotted are 150 ellipticities as measured and compiled by Franx et al. (1991), and another 420 elliptical galaxies observed by Faber et al. (1989). The dissipational merger remnants are nearly indistinguishable from the two samples of observed ellipticals. Both the observed and dissipational ellipticity distributions peak near 0.2 and then drop precipitously above 0.4 . There exist almost no highly elliptical $(\epsilon>0.6)$ galaxies. These features are in sharp contrast to the dissipationless remnants whose distribution of ellipticities peaks near 0.4 and extends beyond 0.7, similar to that found by Naab \& Burkert (2003).

The central velocity dispersion is a common measure of galaxy mass and appears to be correlated with many fundamental properties of elliptical galaxies. As the histogram of remnant velocity dispersions in Figure 3] shows, the central velocity dispersion is highly dependent upon viewing angle, merger orbit, and the dissipative effects of gas. The distribution of dissipationless dispersions is 

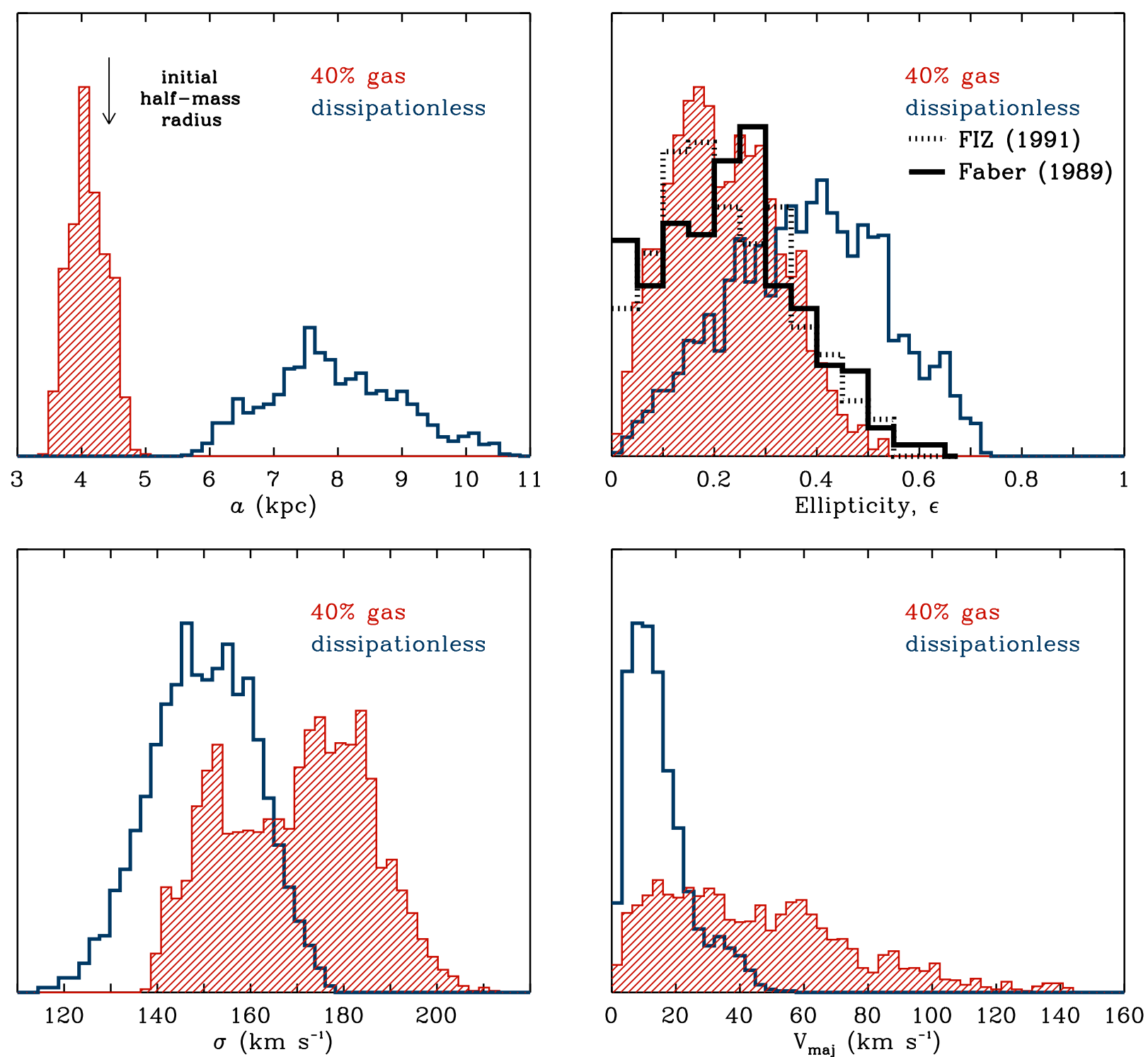

FIG. 3.- Histograms of remnant properties. Shown in (red) cross-hatch are the dissipational remnants, while the open (blue) histogram is for the dissipationless remnants. Histograms show, clockwise from upper-left, the semi-major axis $a$, the half-mass isophote ellipticity $\epsilon$, the central velocity dispersion $\sigma$, and the maximum velocity along the major axis $\mathrm{V}_{\text {maj }}$. Each histogram represents the composite properties of all fifteen remnants, viewed from 150 projections. The semi-major axis histogram includes the half-mass radius of the progenitor disk. The ellipticity histogram includes data on observed ellipticities from Faber et al. (1989) and Franx et al. (1991).

well fitted by a Gaussian with mean $150 \mathrm{~km} \mathrm{~s}^{-1}$ and variance $11 \mathrm{~km} \mathrm{~s}^{-1}$. The distribution of dissipational merger remnants is better described as bi-model, with one component a Gaussian centered at $178 \mathrm{~km} \mathrm{~s}^{-1}$ and variance $10 \mathrm{~km} \mathrm{~s}^{-1}$, and a second component that extends to lower velocity dispersion. This second, lower dispersion, distribution is comprised of five mergers $(d, f, i, l, m)$ that are all significant rotators. The mean and variance of the entire dissipational distribution is $169 \mathrm{~km} \mathrm{~s}^{-1}$ and $15 \mathrm{~km} \mathrm{~s}^{-1}$, respectively.

Because it probes the gravitational potential, the central velocity dispersion is often used in combination with the effective radius as a proxy for galaxy mass (i.e., $\left.M \propto \sigma^{2} R_{e}\right)$. While it does not affect the results of this paper, we note that $\sigma^{2} R_{e}$ for our remnants can vary by a factor of 2 , even though all the remnants galaxies have exactly the same total mass.

The increased velocity dispersion of the dissipational remnants is accompanied by a steeper surface density profile, as shown in Figure 4 Within a radius of $\sim$ $1.8 \mathrm{kpc}$ the dissipational remnants have a much higher surface density owing to gas dissipation and star formation. This feature is present regardless of the merger orbit, even though the different orbits produce a variety of central $(<1 \mathrm{kpc}$ ) profiles. It is intriquing that the surface densities appear to be well fit by a $R^{1 / 4}$-profile and do not demonstrate the distinct cusp of newly formed stars seen in previous simulations (Mihos \& Hernquist 1994a; Springel 2000; Cox et al. 2005). Future work is underway to address whether these surface density profiles are consistent with observed ellipticals and merger remnants (see, e.g., Rothberg \& Joseph 2004; Lauer et al. 2005) as 


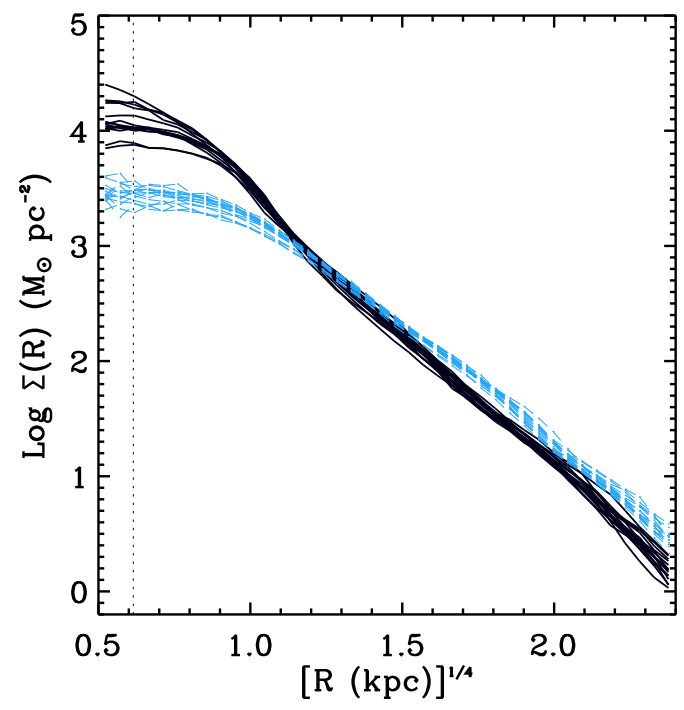

FIG. 4.- The surface density profile for all thirty (the fifteen orientations from Table 1 for both the dissipational and dissipationless mergers) remnants plotted against $R^{1 / 4}$ as viewed from a single projection normal to the orbital plane. The dark solid lines that have uniformly higher central surface density are the dissipational merger remnants. The light dashed lines with lower central surface density of the dissipationless merger remnants. The dashed vertical line denotes the softening length, and hence the resolution of the simulation.

well as whether the profiles depend on progenitor disk gas fraction or mass.

Spheroidal (and possibly all) galaxies are thought to host black holes in their centers in accord with the $M_{\mathrm{BH}}-$ $\sigma$ relation (Ferrarese \& Merritt 2000; Gebhardt et al. 2000). For the remnants displayed here, with central velocity dispersions $\sim 150 \mathrm{~km} \mathrm{~s}^{-1}$, each remnant should contain a fairly massive $\left(\sim\right.$ several $\left.\times 10^{7} \mathrm{M}_{\odot}\right)$ black hole. During the dissipational mergers, black holes grow to the appropriate mass and futher accretion is terminated by a feedback induced galactic wind. Our merger are thus consistent with the idea that the $M_{\mathrm{BH}}-\sigma$ relation is produced by the self-regulated growth of the black hole (Di Matteo et al. 2005; Springel et al. 2005b; Robertson et al. 2006b; Kazantzidis et al. 2005). Because of the lack of gas, the black holes in the dissipationless mergers remain the same size during the entire simulation. In this case, the initial black hole masses require some fine-tuning in order for the remnants to reside on the observed $M_{\mathrm{BH}}-\sigma$ relation. We consider this further evidence for the necessity of gas-rich progenitor disk galaxies.

The final histogram in Figure 3 shows the maximum rotation velocity along the major axis of each remnant. As with the previous histograms, the dissipational and dissipationless remnants are quite different. While the dissipationless remnants rotate very slowly, with all measurements below $50 \mathrm{~km} \mathrm{~s}^{-1}$, the dissipational remnants span a wider range of rotation speeds, with some projections reaching speeds of $\sim 140 \mathrm{~km} \mathrm{~s}^{-1}$. We will discuss the rotational properties of our merger remnants in more detail in the following section.

\subsection{Rotational Support}

As stated in the introduction, elliptical galaxies can be split into two groups. Small ellipticals tend to rotate along their major axes, while large elliptical galaxies show little or no rotation. More specifically, the rotation of small ellipticals is consistent with that of an oblate, isotropic rotator. Thus, their elliptical shape can be understood solely in terms of flattening induced by their observed rotation (Binnev 1978; Binnev \& Tremaine 1987). Large ellipticals, on the other hand, have little rotation. Their elliptical structure must be supported by something other than rotation, and is most likely velocity anisotropy. One of the clearest ways to visualize this trend is to plot the rotation velocity $V_{\text {maj }}$ divided by the central velocity dispersion $\sigma$, against the ellipticity $\epsilon$, in what is commonly referred to as the anisotropy diagram.

Figure 5 shows our merger remnants in an anisotropy diagram. For reference, overplotted on this diagram are data from several observational samples (Davies et al. 1983; Bender 1988; Bender \& Nieto 1990; de Zeeuw et al. 2002). Binnev (1978) has shown that for oblate isotropic rotators $V / \sigma$ is purely a function of ellipticity $\epsilon$. If this is the case, then at high ellipticities one is viewing a rotating, flattened isotropic ellipsoid from edge on. The solid line in Figure [5 is this relation for oblate isotropic rotators

$$
(V / \sigma)=[\epsilon /(1-\epsilon)]^{1 / 2} .
$$

(Binnev 1978). In terms of the anisotropy diagram, galaxies that are near the line are presumably oblate isotropic rotators. Their shapes are flattened by rotation and they are being viewed from a variety of angles with respect to their rotation axes. Galaxies that are below this line, i.e. those that have very little rotation, and some amount of ellipticity, are likely to have strong velocity anisotropies.

As in the previous section, the dissipationless and dissipational remnants occupy very different regions in the anisotropy diagram. The dissipationless remnants, presented in the right-hand plot of Figure 5 occupy a nearly identical region of the anisotropy diagram as those presented in Naab \& Burkert (2003). Consistent with their findings, most remnants are very elliptical and hardly rotating (see also Negroponte \& White 1983; Barnes 1988; Hernquist 1992). We note that our additional orbits $(b-h)$ produce non-rotating remnants exactly like orbits $i-p$ (i.e., the exact orbits simulated by Naab \& Burkert $(2003))$. There are a large number of remnants that have an ellipticity greater than 0.6 , as was noted by Naab \& Burkert (2003), and was clearly displayed in the histogram of Figure 3 This distribution of ellipticities is not observed in real ellipticals, especially for galaxies with little or no rotation. Ellipticity aside, the small rotation of the dissipationless merger remnants led Naab \& Burkert (2003) to suggest dissipationless mergers as a possible formation mechanism for bright ellipticals.

The dissipational merger remnants are shown in the anisotropy diagram on the left panel of Figure 5 These remnants span a much larger range of rotational properties than the dissipational remnants, and include remnants that reside on the oblate isotropic rotator line as well as remnants that fall below this line. The most common position for dissipational remnants to reside in the anisotropy diagram is $V / \sigma \sim 0.35$ and $\epsilon \sim 0.18$. At 

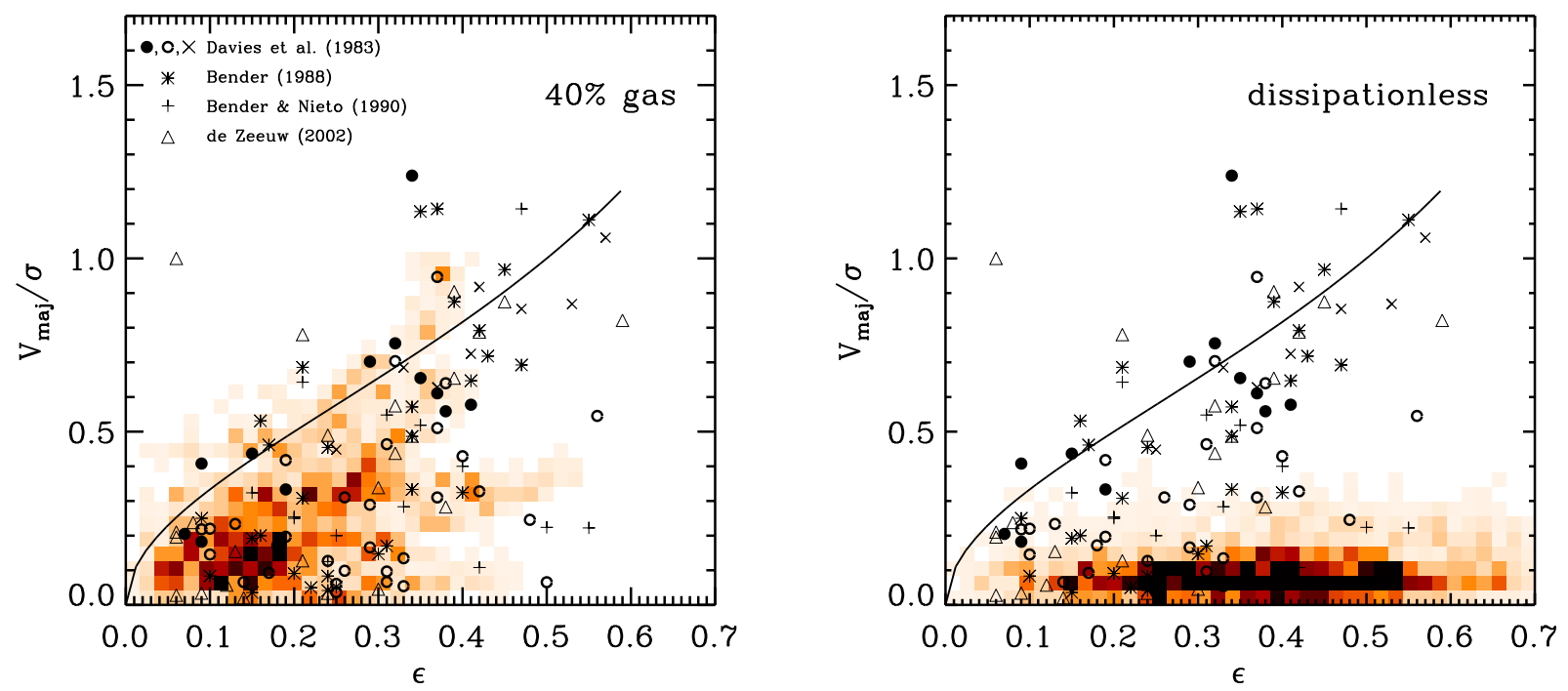

FIG. 5.- The $V_{\mathrm{maj}} / \sigma$ versus ellipticity diagram for dissipational $\left(40 \%\right.$ gas) and dissipationless merger remnants. $V_{\text {maj }}$ is the maximum rotation speed measured in a slit along the major axis, $\sigma$ is the velocity dispersion averaged within half of an half-mass radius, and the ellipticity is measured at the half-mass isophote. Further details can be found in $\S[2.2$. The solid line in both plots is that expected for an oblate isotropic rotator (Binnev 1978). Overplotted are data from observed ellipticals from Davies et al. (1983); Bender (1988); Bender \& Nieto (1990); de Zeeuw et al. (2002).

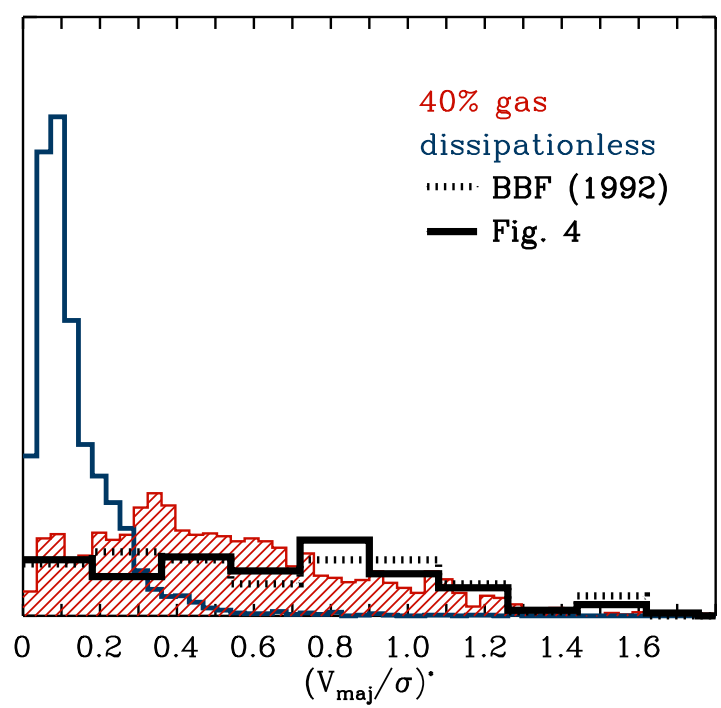

Fig. 6.- Histogram of $\left(\mathrm{V}_{\mathrm{maj}} / \sigma\right)^{*}$ for both the dissipationless (open, blue) and dissipational (filled, red) merger remnants. Also shown is a histogram of observed ellipticals for the same points shown in Figure 5 and a histogram of the sample of spheroids compiled by Bender et al. (1992).

this point the remnant is slowly rotating, is below the oblate isotropic rotator line, and its structure is presumably supported by anisotropy; all of these are features similar to bright ellipticals.

In general, Figure 5 shows that the dissipational remnants encompass a significant fraction of the observational data points; much more so than the dissipationless remnants. To investigate this further, for each one of our merger remnants we evaluate $\left(V_{\text {maj }} / \sigma\right)^{*}$, the ratio of the measured rotation parameter $V_{\text {maj }} / \sigma$ to Equation (1), the value for an isotropic oblate spheroid flattened by rotation (Binnev 1978; Davies et al. 1983; Kormendv \& Bender 1996). In a manner similar to Figure 3 we show histograms of $\left(V_{\mathrm{maj}} / \sigma\right)^{*}$ for both the dissipationless and dissipational merger remnants in Figure 6 In addition we show the distribution for the same observations as shown in Figure 5 as well as the observational sample of Bender et al. (1992). We caution that these samples are not independent of one another as there are a number of galaxies which are counted in both. Confirming the trend in Figure [5] we see that the observations and dissipational remnants have a predominantly flat distribution below $\left(V_{\mathrm{maj}} / \sigma\right)^{*}<1$, and a tail of values above this. In contrast, the dissipational remnants are peaked below 0.2 and there are almost no remnants above 0.6.

Even though the dissipational remnants are a much better match to the observed distribution of $\left(V_{\text {maj }} / \sigma\right)^{*}$, as shown in Figure [6] there is still a discrepancy between the observed and predicted number of fast rotators; i.e., those with $\left(V_{\mathrm{maj}} / \sigma\right)^{*} \geq$ 1. This deficiency was also noted by several other studies under a variety of different circumstances (Cretton et al. 2001; González-García \& Balcells 2005; González-García \& van Albada 2005; Naab \& Burkert 2003), even when minor mergers were included. Observations find a large number of galaxies exhibiting fast rotation. In the observations plotted here, $20 \%$ of the galaxies shown in Figure 5 have $\left(V_{\text {mai }} / \sigma\right)^{*}>1$, and this number increases to $33 \%$ for the Bender et al. (1992) sample which includes a larger number of faint ellipticals and bulges. The fraction of fast rotators could increase even more with the inclusion of S0s and observations which probe the velocity field at large radii (Rix et al. 1999). For our simulated dissipational merger remnants, only $11 \%$ of the projected images have $\left(V_{\mathrm{maj}} / \sigma\right)^{*}>1$. For the moment, we do not find this discrepancy alarming as we have sampled a limited range of disk galaxy initial 


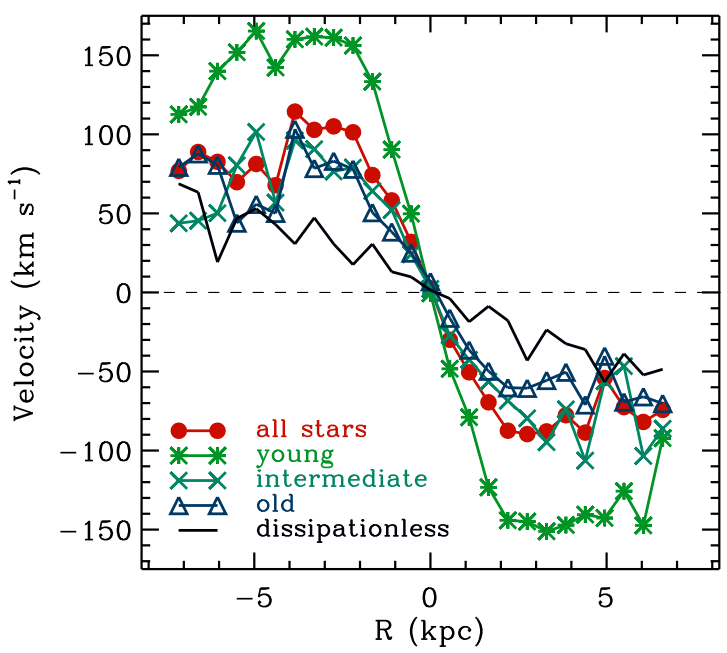

FIG. 7.- The rotation curve for the dissipational merger remnant $f$, viewed perpendicular to the orbital plane. This is one of the fastest rotating remnants and is identical to that used in Figures 1 and 2 The rotation curve is decomposed by particle type according to the key inset within the bottom-left of the plot. The dissipational stellar particles are segregated based upon their relative age; "old" stars begin the simulation as a collisionless particle, "intermediate" stars were created during the isolated and first passage phases, while "young" stars are created from dense gas during the final merger. For reference the composite "all stars" shows the total stellar rotation curve, and the solid black line shows the dissipationless merger remnant viewed from the same angle with an identical slit.

conditions. In particular, we suspect that varying the gas fraction may produce a much larger fraction of fast rotating remnants, continuing the trend seen in Figure 5 We briefly address this in $\S 3.9$

\subsection{The Origin of Stellar Rotation}

To understand what generates the significant rotation found in dissipational remnants, we decompose the stellar rotation curve presented in the lower-left of Figure 11 into contributions from stars by their age. The resulting rotation curves are shown in Figure 7 Star particles are designated as "old", "intermediate", or "young" on the basis of when they originally became a dissipationless stellar particle. Old stars are those that are present prior to the start of the simulations, and thus designated as stars when setting up the initial conditions. Star formation during the course of the merger simulation predominantly occurs in bursts that accompany the first passage of the galaxies, and the final merger event (see e.g., Mihos \& Hernquist 1994c; Cox et al. 2005; Springel et al. 2005b). These burst events occur $\sim 1$ Gyr apart. We denote any stars created during the isolated evolution prior to the first burst, and those generated during the first burst as "intermediate". All stars created during the final merger are "young". In addition to the rotation curves for stars in the dissipational run, we also plot the stars from the dissipationless simulation as a solid black line in Figure 17

Figure 7 demonstrates that it is the stars born during the final merger, i.e., the "young" stars, that are the fastest rotators. Because this young stellar component is centrally concentrated (its half-mass radius is less than $1 \mathrm{kpc}$ ) and is the dominant stellar component inside of $\sim 1 \mathrm{kpc}$, the central rotation closely tracks that of the young stars. Outside of $1 \mathrm{kpc}$ the "intermediate" and "old" populations dominate the stellar mass and thus the rotation curve closely tracks these components. Both "intermediate" and "old" components have similar rotation suggesting that all stars that exist prior to the final merger are equivalent in terms of their rotation properties in the merger remnant.

Figure 7 also plots the rotation curve for the dissipationless version of this merger, shown with a solid black line. While the dissipationless remnant also exhibits rotation, the rotation curve is much shallower than the dissipational remnant. Obviously, gaseous dissipation and star formation have affected the rotation of the old stellar population in the dissipational remnant. The most plausible explanation for this is simply the conservation of angular momentum. The central concentration of young stars steepens the potential well and draws the old stars into the center. The half-mass radius of the "old" stars is $\sim 20 \%$ less than in the dissipational remnant and thus the velocity of these stars must be roughly $\sim 20 \%$ higher to conserve angular momentum. The fact that the velocity increase is larger than this may represent a transfer of angular momentum between the stars and gas, as was shown by Mihos \& Hernquist (1996).

Even though the young stars rotate faster than the old stars, most of the angular momentum is carried by the old stars. Specifically, the old stars typically have three times the specific angular momentum of the young stars. However, most of the angular momentum carried by the old stars is at radii larger than the effective radius, and thus is not contained in our slit which only extends slightly beyond one effective radius. Figure $\mathbf{7}$ shows that beyond $\sim 4 \mathrm{kpc}$, the dissipational and dissipationless remnants have similar rotation, and also similar angular momentum distributions for the old stellar component. Thus, these results suggest that the significant rotation seen in dissipational remnants is not indicative of more angular momentum, but dissipation and star formation effectively redistribute angular momentum from large radii to within the effective radius where rotation is typically measured.

Although our results indicate a segregation between stellar age and rotation it is unclear if this trend could be verified by observations. As the stellar populations evolve, the small age differences (1-2 Gyr) will quickly be washed out and thus difficult to disentangle. However, the general trend for young stars to be centrally concentrated and kinematically different than the more extended older stars is a generic feature of these simulations. In a few cases the merger results in counter-rotating or kinematically decoupled cores, as is seen in many observed ellipticals (Davies \& Birkinshaw 1988; Franx et al. 1989), and as found earlier by Hernquist \& Barnes (1991) using simulations with a smaller gas fraction than here and ignoring star formation.

\subsection{Individual Merger Remnants}

Although dissipational remnants display much more rotation, on average, than their dissipationless counterparts, there is a large spread of rotation properties be- 
TABLE 2

Projection Averaged Remnant Properties

\begin{tabular}{|c|c|c|c|c|c|c|c|c|c|c|c|c|}
\hline $\begin{array}{c}\text { Orientation } \\
\text { ID }\end{array}$ & $\begin{array}{c}\epsilon \\
(1)\end{array}$ & $\begin{array}{c}\mathrm{R}_{e} \\
(\mathrm{kpc}) \\
(2)\end{array}$ & $\begin{array}{c}a \\
(\mathrm{kpc}) \\
(3)\end{array}$ & $\begin{array}{c}\text { Dissipation } \\
V_{\mathrm{maj}} \\
\left(\mathrm{km} \mathrm{s}^{-1}\right) \\
(4)\end{array}$ & $\begin{array}{c}\sigma \\
\left(\mathrm{km} \mathrm{s}_{(5)} \mathrm{s}^{-1}\right)\end{array}$ & $\begin{array}{c}\left(V_{\mathrm{maj}} / \sigma\right)^{*} \\
(6)\end{array}$ & $\begin{array}{c}\epsilon \\
(1)\end{array}$ & $\begin{array}{c}\mathrm{R}_{e} \\
(\mathrm{kpc}) \\
(2)\end{array}$ & $\begin{array}{c}a \\
(\mathrm{kpc}) \\
(3)\end{array}$ & $\begin{array}{c}40 \% \mathrm{Gas} \\
V_{\mathrm{maj}} \\
\left(\mathrm{km} \mathrm{s}^{-1}\right) \\
(4)\end{array}$ & $\begin{array}{c}\sigma \\
\left(\mathrm{km} \mathrm{s}^{-1}\right) \\
(5)\end{array}$ & $\begin{array}{c}\left(V_{\mathrm{maj}} / \sigma\right)^{*} \\
(6)\end{array}$ \\
\hline $\mathrm{h}$ & 0.48 & 6.49 & 8.73 & 14.3 & 150.8 & 0.09 & 0.27 & 3.56 & 4.16 & 46.0 & 180.7 & 0.25 \\
\hline b & 0.46 & 6.27 & 8.21 & 11.7 & 150.0 & 0.08 & 0.27 & 3.70 & 4.21 & 26.8 & 182.2 & 0.15 \\
\hline $\mathrm{c}$ & 0.50 & 6.37 & 8.39 & 15.8 & 153.5 & 0.10 & 0.32 & 3.70 & 4.43 & 116.6 & 177.5 & 0.65 \\
\hline d & 0.42 & 6.40 & 8.04 & 9.1 & 151.0 & 0.06 & 0.24 & 3.86 & 4.34 & 111.9 & 147.7 & 0.76 \\
\hline $\mathrm{e}$ & 0.37 & 6.23 & 7.87 & 9.7 & 149.7 & 0.06 & 0.21 & 3.49 & 3.99 & 11.3 & 178.5 & 0.06 \\
\hline $\mathrm{f}$ & 0.33 & 6.16 & 7.17 & 15.3 & 147.0 & 0.10 & 0.26 & 3.99 & 4.54 & 143.3 & 140.5 & 1.03 \\
\hline $\mathrm{g}$ & 0.37 & 6.19 & 7.60 & 13.0 & 150.2 & 0.09 & 0.19 & 3.84 & 4.19 & 65.5 & 172.7 & 0.37 \\
\hline $\mathrm{i}$ & 0.34 & 6.24 & 7.71 & 13.8 & 150.4 & 0.09 & 0.19 & 3.64 & 4.00 & 115.7 & 155.4 & 0.75 \\
\hline $\mathrm{j}$ & 0.22 & 6.09 & 6.91 & 15.5 & 149.6 & 0.10 & 0.13 & 3.36 & 3.63 & 66.0 & 169.6 & 0.39 \\
\hline $\mathrm{k}$ & 0.49 & 6.39 & 8.83 & 9.3 & 151.4 & 0.06 & 0.22 & 3.56 & 4.01 & 73.2 & 175.2 & 0.41 \\
\hline 1 & 0.30 & 6.24 & 7.49 & 27.2 & 147.8 & 0.18 & 0.19 & 4.12 & 4.49 & 91.5 & 147.8 & 0.62 \\
\hline $\mathrm{m}$ & 0.39 & 6.21 & 7.99 & 14.0 & 151.4 & 0.09 & 0.17 & 4.01 & 4.30 & 77.2 & 146.2 & 0.53 \\
\hline $\mathrm{n}$ & 0.26 & 6.29 & 7.34 & 12.3 & 150.4 & 0.08 & 0.21 & 3.50 & 3.93 & 131.4 & 169.4 & 0.77 \\
\hline o & 0.35 & 6.20 & 7.46 & 31.2 & 146.6 & 0.21 & 0.20 & 3.67 & 4.11 & 128.7 & 166.4 & 0.77 \\
\hline p & 0.45 & 6.29 & 8.21 & 8.0 & 152.1 & 0.05 & 0.24 & 3.59 & 4.07 & 66.0 & 168.6 & 0.39 \\
\hline
\end{tabular}

Note. - List of properties of simulated merger remnants averaged from 190 viewing angles selected to uniformly sample the unit sphere. Column (1) is the ellipticity of the half-mass isophote. Column (2) is the half-mass radius as measured using circular apertures. Column (3) is semi-major axis of the half-mass isophote. Column (4) is the maximum rotation speed along the major axis. Column (5) is the central velocity dispersion, averaged within $0.5 a$, and column (6) is the ratio of $V_{\text {maj }} / \sigma$ to that expected from an oblate isotropic rotator.

tween individual remnants (Bendo \& Barnes 2000). Figure 8 demonstrates this behavior by displaying the rotational properties of individual merger remnants, one per panel, viewed from 190 projections. It is these remnants that are co-added to produce Figure 5

Figure 8 clearly shows that projection effects cause each remnant to occupy a band in the anisotropy diagram. Every remnant appears nearly circular from a small range of viewing angles and elliptical from most viewing angles. It is typically the case that remnants rotate very little when they appear circular and thus each band passes near the origin of the anisotropy diagram. At larger projected ellipticities there exists a wide range of rotational properties for the fifteen merger remnants. In some cases $(d, f, i$, and $o$ ) the remnant closely resembles an oblate isotropic rotator. However, in most cases the remnant swath is below the oblate isotropic rotator line. In all cases (except, perhaps, the $j$ merger remnant) the maximum rotation is coincident with the largest projected ellipticity, suggesting that the majority of the dissipational merger remnants are (nearly) oblate and the rotation axis is along their short axis.

It is important to emphasize the large range of kinematic properties present in the dissipational merger remnants. This range of remnant properties owes entirely to the variations in initial disk orientations. Specifically, it appears that the co-planar orbits $(h, b$, and $c)$ produce very flattened anisotropic remnants, while polar orbits, i.e., where one disk orbits in a $90^{\circ}$ orientation to another disk $(d$ and $f)$, result in fast rotating remnants that are oblate and isotropic. Finally, tilted orbits, such as $e, j$, and $k$ produce fairly round remnants that are not rotating.

The dissipationless merger remnants shown in Figure 8 show much less variability than the dissipational remnants. In all cases, the remnants span a wide range of ellipticities and are never rotating. Elliptical remnants that do not rotate are thought to owe their ellipsoidal shape to anisotropic velocity dispersions. We will investigate this in the following section.

A final point concerns the initial disk orientations and whether or not they faithfully represent the full range of possibilities. Orientations $i-p$ were originally selected to be a uniform sampling of disk orientations (see Barnes 1992). However, as we note above, the co-planar orbits $(h, b$, and $c)$ and polar orbits $(d$ and $f)$ produce somewhat peculiar remnants that are not well represented by the uniform sampling introduced by Barnes. Hence, it seems that one must include both a uniform grid to represents the majority of merger events as well as a small sample of hand-selected orientations to represent more pathological orbits in order to gain a full understanding of the various properties possible in the aftermath of a galaxy collision.

\subsection{Anisotropy}

In the previous section, we demonstrated that all of the dissipationless and several of the dissipational merger remnants are ellipsoidal and slowly rotating. In short, they reside below the oblate isotropic rotator line in the anisotropy diagram. In this case, it is assumed that the ellipsoidal shapes are supported by an anisotropic velocity dispersion. However, it has recently been shown that mergers between unequal mass galaxies can produce fast rotators that are very anisotropic (Burkert \& Naab 2005), and thus we wonder if the slowly rotating remnants are necessarily anisotropic. In this section we attempt to address this question by measuring the velocity anisotropy of each merger remnant.

We note that any dissipationless system in equilibrium can be accurately described by the tensor virial theorem

$$
2 T_{j k}+\Pi_{j k}=-W_{j k},
$$

where $W_{j k}$ is the potential energy tensor, and $T_{j k}$ and $\Pi_{j k}$ are the ordered and random components, respectively, of the kinetic energy tensor (Binnev \& Tremaine 

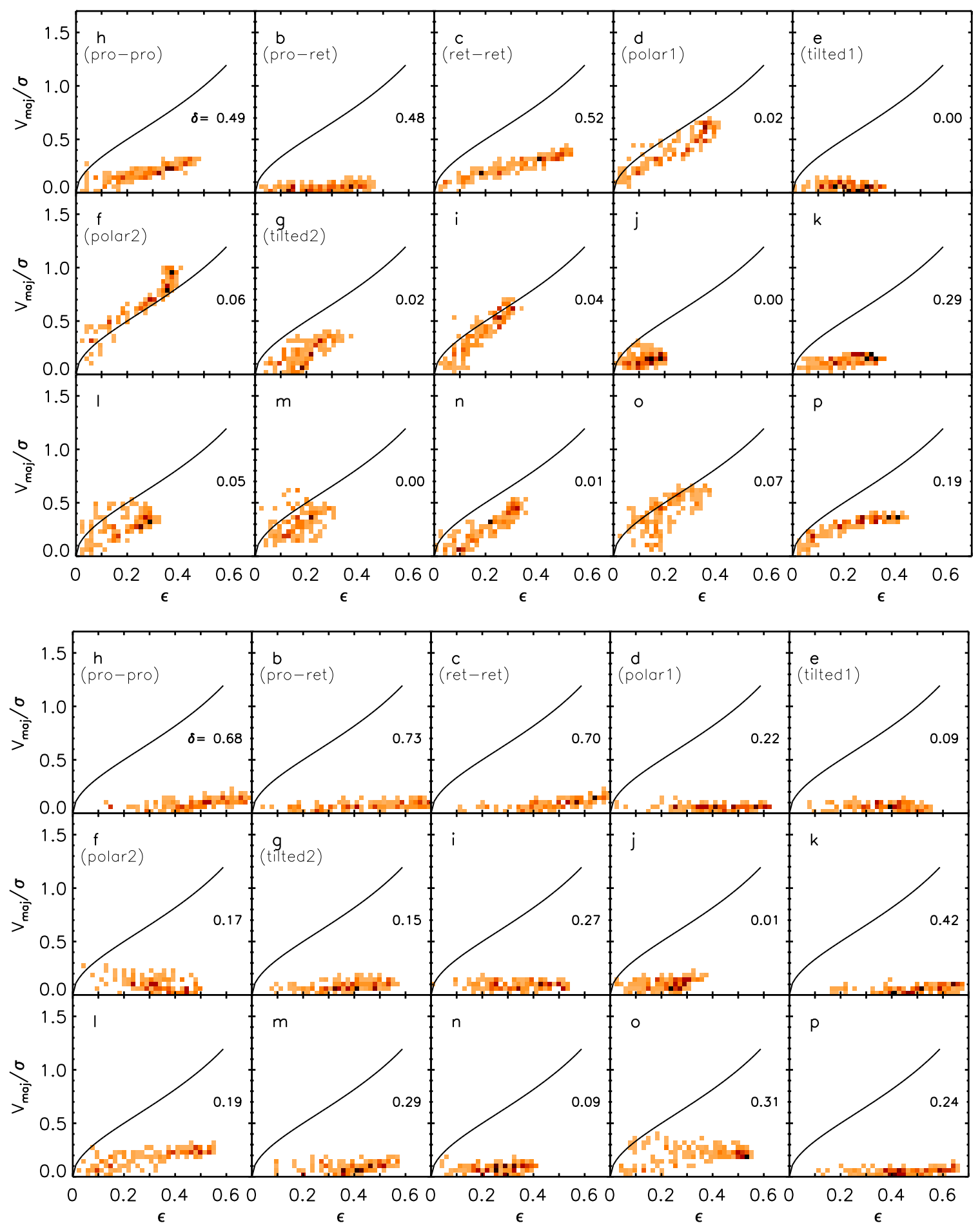

FIG. 8.- $V_{\text {maj }} / \sigma$ versus ellipticity, or anisotropy, diagram for individual merger remnants. The top grid of fifteen panels shows remnants where the progenitor disk contained $40 \%$ gas and the bottom grid is the corresponding mergers when the progenitor disks are dissipationless. Each plot shows one merger remnant for one of the merger orientations listed in Table 1 viewed from 190 different viewing angles chosen to uniformly sample the unit sphere. The solid line is that expected for an oblate isotropic rotator and the number in the middle-right of each panel is the anisotropy parameter $\delta$ defined in $\S$ 3.5 For reference, $\delta=0$ is isotropic and $\delta>0$ is a sign of anisotropy.

1987, eq. 4-78). Taking the trace of Equation (2) results in the scalar virial theorem, which simply relates the total kinetic energy to the total potential energy.

To calculate the kinetic energy tensor we assume each N-body stellar particle has a gaussian velocity distribu- tion. The mean and distribution are computed by kernel weighting among the 96 nearest neighbors. A Cartesian coordinate system is selected such that the $\mathrm{z}$-axis is parallel to the original orbital angular momentum and the $\mathrm{x}$ and $\mathrm{y}$ axes are arbitrary. The mean velocity then con- 


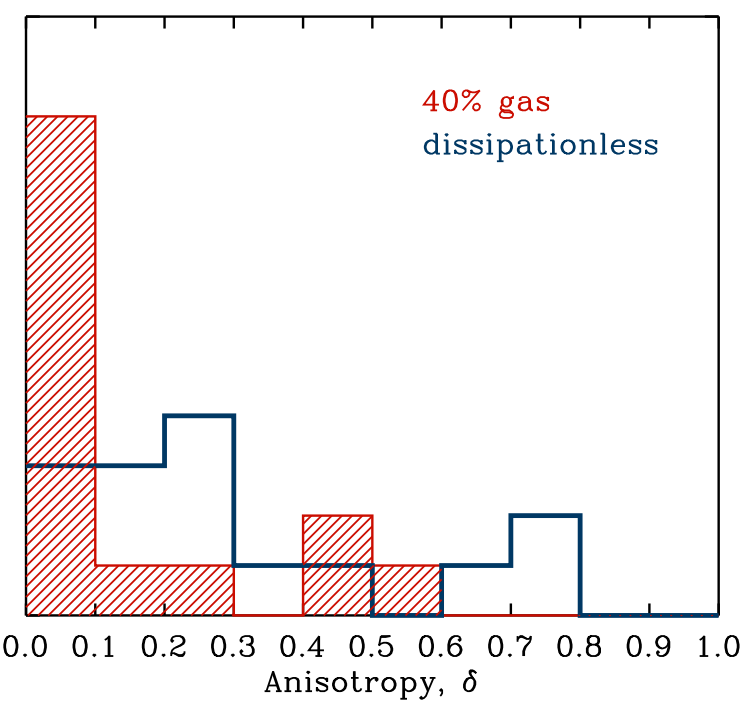

FIG. 9.- Histogram of remnant velocity anisotropy parameters $\delta$, where $\delta$ is calculated via Equation (4). With this definition for anisotropy, $\delta=0$ is isotropic and $\delta>0$ is anisotropic.

tributes to $T_{j k}$ and the dispersion to $\Pi_{j k}$. For our merger remnants the kinetic energy is predominantly in the form of random motions, with

$$
\frac{\operatorname{Trace}\left(\Pi_{j k}\right)}{\operatorname{Trace}\left(T_{j k}\right)} \geq 3 \text {. }
$$

In comparison, this ratio for the progenitor disks is $\sim$ 0.17 .

The anisotropy of each remnant, then, is the degree to which the elements of $\Pi_{j k}$, the velocity dispersion tensor, are unequal. Owing to our initial orbital configuration, in which the orbital angular momentum is aligned with the z-axis, we typically find $\Pi_{x x}, \Pi_{y y}>\Pi_{z z}$. We follow Burkert \& Naab (2005) and define the anisotropy of the system as

$$
\delta=1-\frac{2 \Pi_{\mathrm{zz}}}{\Pi_{\mathrm{xx}}+\Pi_{\mathrm{yy}}} .
$$

With this definition, an isotropic system has $\delta=0$, and $\delta>0$ indicates some degree of anisotropy. For two of the dissipational remnants ( $g$ and $n), \delta$ is slightly negative. However, these remnants are nearly isotropic and in this case we quote the absolute value of $\delta$.

A histogram of remnant anisotropies is presented in Figure 9 and the anisotropy of individual merger remnants is printed in each panel of Figure 8 along with the swath of $V_{\text {maj }} / \sigma$ owing to various projections. From Figure 9 we see a systematic decrease in $\delta$ for dissipational remnants compared to dissipationless remnants. In other words, every dissipational remnant is much closer to isotropic than its dissipationless counterpart. Roughly two-thirds $(9 / 15)$ of the dissipational remnants are consistent with being isotropic $(\delta<0.05)$ compared to only one of the dissipationless remnants $(j)$. Although there is a fair amount of scatter, there seems to be a uniform change in the anisotropy for dissipational mergers equivalent to $\Delta \delta \sim-0.16$ compared to dissipationless remnants.

As with the rotation, Figure 8 also shows that the initial disk orientations have a large effect on the remnant anisotropy. This fact is perhaps not that surprising given the strong link between rotation and anisotropy. In particular, the three initial orientations in which the initial disks are aligned with the orbital plane, i.e., co-planar mergers, $(h, b$, and $c)$ result in remnants that are much more anisotropic than any of the other orientations. This trend appears to hold regardless of the presence of gas or not.

Figure 8 also allows us to assess the relation between our (rough) measure of anisotropy $\delta$ and rotation, as measured by the anisotropy diagram. As outlined in $\S 3.2$ these quantities should be anti-correlated, so that fast rotators are likely to be isotropic, while flattened non-rotators should be anisotropic. However, this relation has not been tested in numerical experiments such as ours.

At first glance, we note that the three dissipational remnants $(d, f$, and $i$ ) whose projected rotation is consistent with the line for oblate isotropic rotators (the solid line in the anisotropy diagram) are also isotropic $(\delta<0.05)$. Next, many of the remnants that show very little projected rotation (dissipational remnants $h$, $b, c, k, p$ and every dissipationless remnant except $j$ ) are all anisotropic. Therefore, for at least 22 of the 30 total merger remnants, the expected relation between anisotropy and rotation appears to hold.

However, there remain eight remnants for which their rotation properties are difficult to reconcile with their anisotropy. Of these eight, only one dissipationless remnant seems out of place; $j$. This merger remnant is not rotating and is moderately flattened (average ellipticity of 0.22 ) yet is apparently isotropic. One of the dissipational remnants, $e$, is similar. The six remaining outlyers are all dissipational remnants $(g, j, l, m, n$, and $o$ ) and are all quite similar. These remnants are round (average ellipticity $\approx 0.18$ ), and have a large range of rotational properties for any one projected ellipticity. As such these remnants appear to occupy more extended regions within the anisotropy diagram, rather than distinct swathes. Future work will be necessary to determine whether the measure of anisotropy provided by equation (4) is sufficient to describe these systems as well as to determine what generates their anomalous properties.

\subsection{Shapes}

The results presented in $\S 3.1$ specifically the distribution of projected ellipticities in Figure 3 show that, in projection, dissipational remnants tend to be rounder than dissipationless remnants. To further quantify this trend we determine the three-dimensional shape of each remnant as measured by the axis ratios of the inertia tensor (Barnes 1992; Hernquist 1992, 1993; Springel 2000). Because our analysis has focused upon the stellar component, primarily within $R_{e}$, we use only the most bound half of all stellar particles to calculate the inertia tensor. We note that there are many other methods by which the shapes can be quantified. Most of these methods calculate the eigenvectors from some form of the inertia tensor. In our case, we calculated the inertia tensor based upon the most bound stellar particles but alternatives are spherical or ellipsoidal windows which may be interatively adjusted until the measured shape and selected aperture agree (see e.g., Allgood et al. 2006, for survey of different methods and recent results from the 
TABLE 3

REMNANT Shapes

\begin{tabular}{l|ccc|ccc}
\hline \hline & \multicolumn{3}{|c}{ Dissipationless } & \multicolumn{3}{c}{$40 \%$ Gas } \\
ID & $b$ & $c$ & $T$ & $b$ & $c$ & $T$ \\
\hline $\mathrm{h}$ & 0.73 & 0.42 & 0.56 & 0.94 & 0.43 & 0.14 \\
$\mathrm{~b}$ & 0.86 & 0.39 & 0.30 & 0.94 & 0.42 & 0.14 \\
$\mathrm{c}$ & 0.75 & 0.37 & 0.50 & 0.98 & 0.40 & 0.04 \\
$\mathrm{~d}$ & 0.77 & 0.55 & 0.58 & 0.90 & 0.67 & 0.34 \\
$\mathrm{e}$ & 0.76 & 0.62 & 0.68 & 0.85 & 0.71 & 0.57 \\
$\mathrm{f}$ & 0.78 & 0.67 & 0.67 & 0.94 & 0.40 & 0.41 \\
$\mathrm{~g}$ & 0.79 & 0.62 & 0.61 & 0.86 & 0.74 & 0.57 \\
$\mathrm{i}$ & 0.82 & 0.60 & 0.52 & 0.93 & 0.69 & 0.24 \\
$\mathrm{j}$ & 0.84 & 0.76 & 0.73 & 0.96 & 0.82 & 0.24 \\
$\mathrm{k}$ & 0.69 & 0.49 & 0.69 & 0.93 & 0.62 & 0.21 \\
$\mathrm{l}$ & 0.91 & 0.61 & 0.27 & 0.93 & 0.71 & 0.28 \\
$\mathrm{~m}$ & 0.72 & 0.60 & 0.75 & 0.88 & 0.71 & 0.44 \\
$\mathrm{n}$ & 0.81 & 0.73 & 0.74 & 0.97 & 0.81 & 0.15 \\
$\mathrm{o}$ & 0.96 & 0.62 & 0.12 & 0.96 & 0.79 & 0.19 \\
$\mathrm{p}$ & 0.76 & 0.52 & 0.58 & 0.93 & 0.63 & 0.23 \\
& & & & & &
\end{tabular}

Note. - List of remnant axial ratios $b$ and $c$ and triaxiality parameter $T$, as defined in $\S 3.6$

literature).

The axis ratios $b$ and $c$ are defined as $b=\left(\lambda_{2} / \lambda_{1}\right)^{1 / 2}$ and $c=\left(\lambda_{3} / \lambda_{1}\right)^{1 / 2}$, where $\lambda_{1} \geq \lambda_{2} \geq \lambda_{3}$ are the eigenvalues of the inertia tensor. Values of $b, c$, along with the "triaxiality" parameter $T=\left(1-b^{2}\right) /\left(1-c^{2}\right)$ (Franx et al. 1991) are listed in Table 3 for all of the dissipationless and dissipational remnants. For reference, $T=1$ is prolate, and $T=0$ is oblate, and all values in between are triaxial.

While Table 3 is useful for assessing the effects of dissipation for any particular merger, the overall trends are more apparent if one plots the axial ratio $a$ against $b$ as is done in Figure 10, or a histogram of the triaxiality parameter $T$ as in Figure 11 Both of these figures show that dissipationless remnants are triaxial with a tendency to be more prolate. This result is also apparent in previous studies of dissipationless mergers (Barnes 1992; Hernquist 1992; Springel 2000).

Figures 10]and 11]demonstrate that dissipational remnants are also triaxial, however these remnants tend to be much closer to oblate. This result is consistent with previous simulations that contained 20\% gas (Springel 2000), or a dense stellar bulge (Hernquist 1993; Springe] 2000; González-García \& Balcells 2005). It is noteworthy that the three dissipational systems that appear to be oblate isotropic rotators $(d, f$, and $i)$ from their swath on the anisotropy diagram are actually quite triaxial ( $T=0.34,0.41$, and 0.24 , respectively). However, the shape of merger remnants is known to be a function of radius (Barnes 1992; Hernquist 1992, 1993), and by simply repeating our analysis on particles which are progressively more bound, we find that the central regions of the remnants are much more oblate than the outer regions. Therefore, the apparent discrepancy between the rotation of the remnant and its shape is likely a byproduct of our selecting the most bound half of stellar particles rather than a genuine sign of anomalous kinematics. In $\S 3.9$ we investigate how our measure of the triaxiality depends on orbit, gas fraction and progenitor mass.

Because real ellipticals can be observed from only a single projection, their intrinsic shapes are difficult to assess. From the distribution of observed axial ratios, several works have shown that elliptical galaxies can not be entirely composed of oblate (Rvden 1992; Lambas et al. 1992; Tremblav \& Merritt 1995; Alam \& Rvden 2002) or prolate (Tremblav \& Merritt 1995) spheroids. Merritt \& Tremblav (1996) presented intriquing evidence that there exists a bimodal distribution of shapes, namely low luminosity ellipticals are consistent with being oblate spheroids, while bright elliticals are only consistent with a triaxial intrinsic shape that is rounder on average than low-luminosity elliticals. A similar conclusion was reached by Franx et al. (1991) from analysis of a much smaller set of ellipticals that also contained kinematic information. In their study, the triaxial shapes are necessary to match the small percentage of galaxies with rotation observed along their photometric minor axis (see $\S \mathbf{3 . 7}$ for additional details) and we note that their mean triaxiality parameter $T$ was less than 0.4. We find that the mean triaxiality parameter for the dissipational remnants is 0.28 as opposed to 0.55 for the dissipationless remnants.

\subsection{Minor Axis Rotation}

A number of elliptical galaxies are observed to have rotation along their minor axes (see e.g., Franx et al. 1991, and references therein). The presence of minor axis rotation is possible in a triaxial or prolate ellipsoid and impossible in an oblate, axisymmetric one (Binnev 1985; de Zeeuw \& Franx 1991). The previous section demonstrated that all of our merger remnants show some degree of triaxiality and hence it is possible that any, or all, or our remnants could have some degree of minor axis rotation. However, because the dissipationless remnants are much more prolate (and definitely more triaxial) than dissipational ones, it is likely that dissipationless remnants will have more minor axis rotation.

In order to determine the degree of minor axis rotation, we repeat the analysis outlined in $\S 2.2$ with the slit now placed along the minor axis of each remnant. Thus, for each remnant we have a rotation speed along the major $\left(V_{\mathrm{maj}}\right)$ and minor $\left(V_{\mathrm{min}}\right)$ axes. We list the minor axis rotation for each of our merger remnants in Table 4 As an additional measure of the minor axis rotation we follow Binnev (1985) and measure

$$
\mu=\frac{V_{\text {min }}}{\left(V_{\text {maj }}^{2}+V_{\text {min }}^{2}\right)^{1 / 2}},
$$

the minor axis rotation parameter. An additional measure of the minor axis rotation, and one that is useful because it can be compared directly to observations, is the kinematic misalignment $\Psi$, defined by

$$
\tan \Psi=\frac{V_{\mathrm{min}}}{V_{\mathrm{maj}}} .
$$

Both minor axis parameters, $\mu$ and $\Psi$, as well as the minor axis rotation speed $V_{\text {min }}$, are listed in Table 4 for each merger remnant.

Surprisingly, Table 4 shows that the dissipationless and dissipational remnants have nearly identical minor axis rotation speeds. However, because the major axis rotation is much larger for the dissipational remnants, both $\mu$ and $\Psi$ are much smaller for the dissipational merger remnants. 

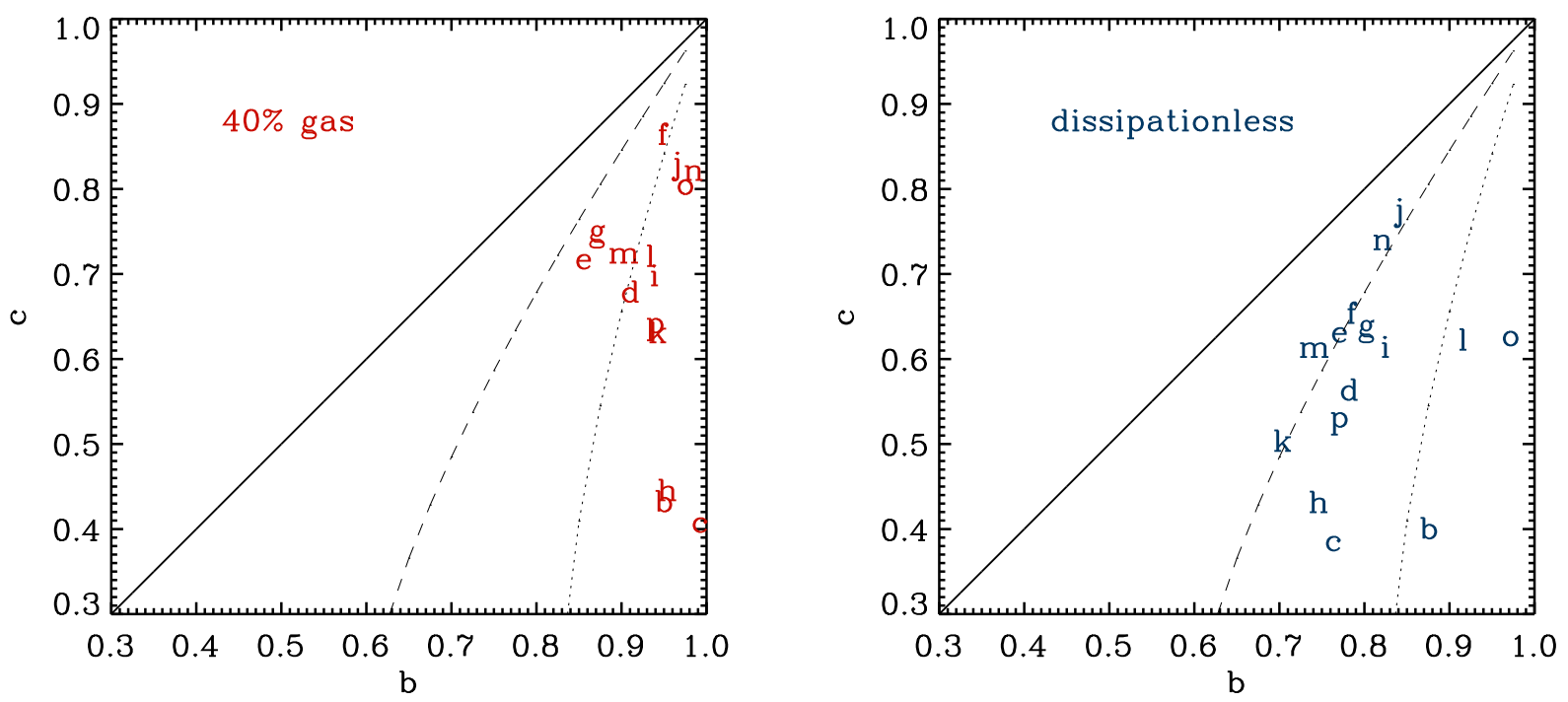

FIG. 10. - The shape diagram for merger remnants. $b$ and $c$ are defined as $\left(\lambda_{2} / \lambda_{1}\right)^{1 / 2}$, and $\left(\lambda_{3} / \lambda_{1}\right)^{1 / 2}$, respectively. $\lambda_{1}, \lambda_{2}$, and $\lambda_{3}$ are the eigenvalues of the inertia tensor in ascending order. Only the most bound half of particles are used when computing the inertia tensor. The solid line which denotes $c=b$ would be a prolate spheroid with $T=1$, where $T=\left(1-b^{2}\right) /\left(1-c^{2}\right)$ is the triaxiality parameter. The right vertical axis, at $b=1$ would be an oblate spheroid with $T=0$. The dashed line shows $T=2 / 3$, and the dotted line shows $T=1 / 3$.

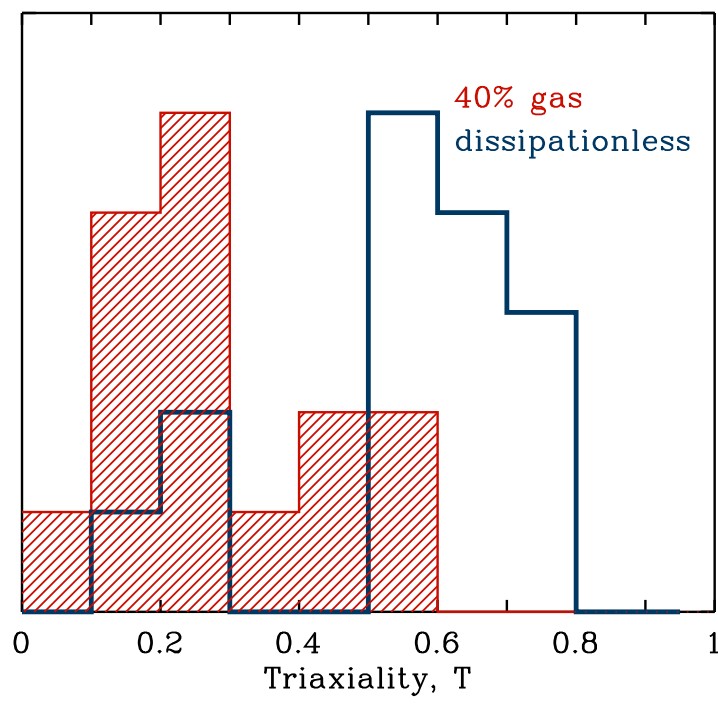

Fig. 11.- Histogram of remnant "triaxiality" parameters $T$, where $T=\left(1-b^{2}\right) /\left(1-c^{2}\right)$, as defined by Franx et al. (1991). Oblate galaxies have $T=0$, prolate galaxies have $T=1$, and all values in between, as the remnants presented here, are triaxial.

Figure 12 presents another way to visualize the relation between minor and major axis rotation by plotting the minor axis rotation parameter $\mu$ against the "total" rotation speed $V_{\text {tot }}=\left(V_{\text {maj }}^{2}+V_{\text {min }}^{2}\right)^{1 / 2}$. All merger remnants inhabit a clear band in the $\mu-V_{\text {tot }}$ plane. However, the dissipational and dissipationless remnants are concentrated in very different regions. Fast rotators, those with $V_{\text {tot }}>50 \mathrm{~km} \mathrm{~s}^{-1}$, have very little minor axis rotation and are produced solely by dissipational mergers. Slow rotators, those with total rotation speeds below $50 \mathrm{~km} \mathrm{~s}^{-1}$ have a wide variety of minor axis rotation parameters. Also, there is a clear preference for dissipa-
TABLE 4

Minor Axis Kinematics

\begin{tabular}{|c|c|c|c|c|c|c|}
\hline \multirow[b]{2}{*}{ ID } & \multicolumn{3}{|c|}{ Dissipationless } & \multicolumn{3}{|c|}{$40 \%$ Gas } \\
\hline & $\begin{array}{c}V_{\min } \\
\left(\mathrm{km} \mathrm{s}^{-1}\right)\end{array}$ & $\mu$ & $\Psi$ & $\begin{array}{c}\mathrm{V}_{\min } \\
\left(\mathrm{km} \mathrm{s}^{-1}\right)\end{array}$ & $\mu$ & $\Psi$ \\
\hline $\mathrm{h}$ & 11.2 & 0.59 & 39.3 & 11.3 & 0.30 & 18.1 \\
\hline $\mathrm{b}$ & 10.6 & 0.64 & 41.9 & 8.2 & 0.37 & 23.0 \\
\hline $\mathrm{c}$ & 10.0 & 0.52 & 33.1 & 8.3 & 0.10 & 6.2 \\
\hline $\mathrm{d}$ & 30.1 & 0.93 & 71.1 & 29.2 & 0.29 & 18.0 \\
\hline $\mathrm{e}$ & 14.9 & 0.78 & 55.0 & 12.6 & 0.70 & 47.3 \\
\hline $\mathrm{f}$ & 66.9 & 0.93 & 74.5 & 32.8 & 0.23 & 13.7 \\
\hline $\mathrm{g}$ & 14.5 & 0.70 & 47.3 & 14.4 & 0.31 & 20.7 \\
\hline $\mathrm{i}$ & 43.6 & 0.90 & 68.3 & 31.1 & 0.31 & 19.3 \\
\hline $\mathrm{j}$ & 22.9 & 0.77 & 53.8 & 34.9 & 0.50 & 32.3 \\
\hline $\mathrm{k}$ & 19.1 & 0.87 & 63.5 & 19.5 & 0.32 & 19.8 \\
\hline l & 44.6 & 0.82 & 57.9 & 52.7 & 0.51 & 32.3 \\
\hline $\mathrm{m}$ & 52.6 & 0.94 & 73.8 & 54.9 & 0.57 & 37.0 \\
\hline $\mathrm{n}$ & 35.6 & 0.91 & 68.8 & 37.1 & 0.33 & 20.4 \\
\hline 0 & 40.0 & 0.75 & 51.1 & 40.3 & 0.36 & 23.0 \\
\hline $\mathrm{p}$ & 32.1 & 0.94 & 74.0 & 22.6 & 0.34 & 20.6 \\
\hline
\end{tabular}

NoTE. - Minor axis rotation properties of the simulated merger remnants. $V_{\min }$ is the minor axis rotation speed, in $\mathrm{km} \mathrm{s}^{-1}, \mu$ is the minor axis rotation parameter, defined by Equation $[5$ and $\Psi$ is the kinematic misalignment defined by Equation 6

tionless merger remnants to have significant minor axis rotation relative to the total rotation. In fact, the majority of the dissipationless merger remnants have more minor axis rotation than major axis rotation.

To confirm the large difference between the minor axis kinematics of dissipationless versus dissipational merger remnants, we show the histograms of the kinematic misalignment parameter $\Psi$ from all projections in Figure 13 Again, a clear dichotomy in $\Psi$ is shown. Dissipationless remnants have significant minor axis rotation and thus large kinematic misalignments. Overplotted are data compiled by Franx et al. (1991) shown with a thick 

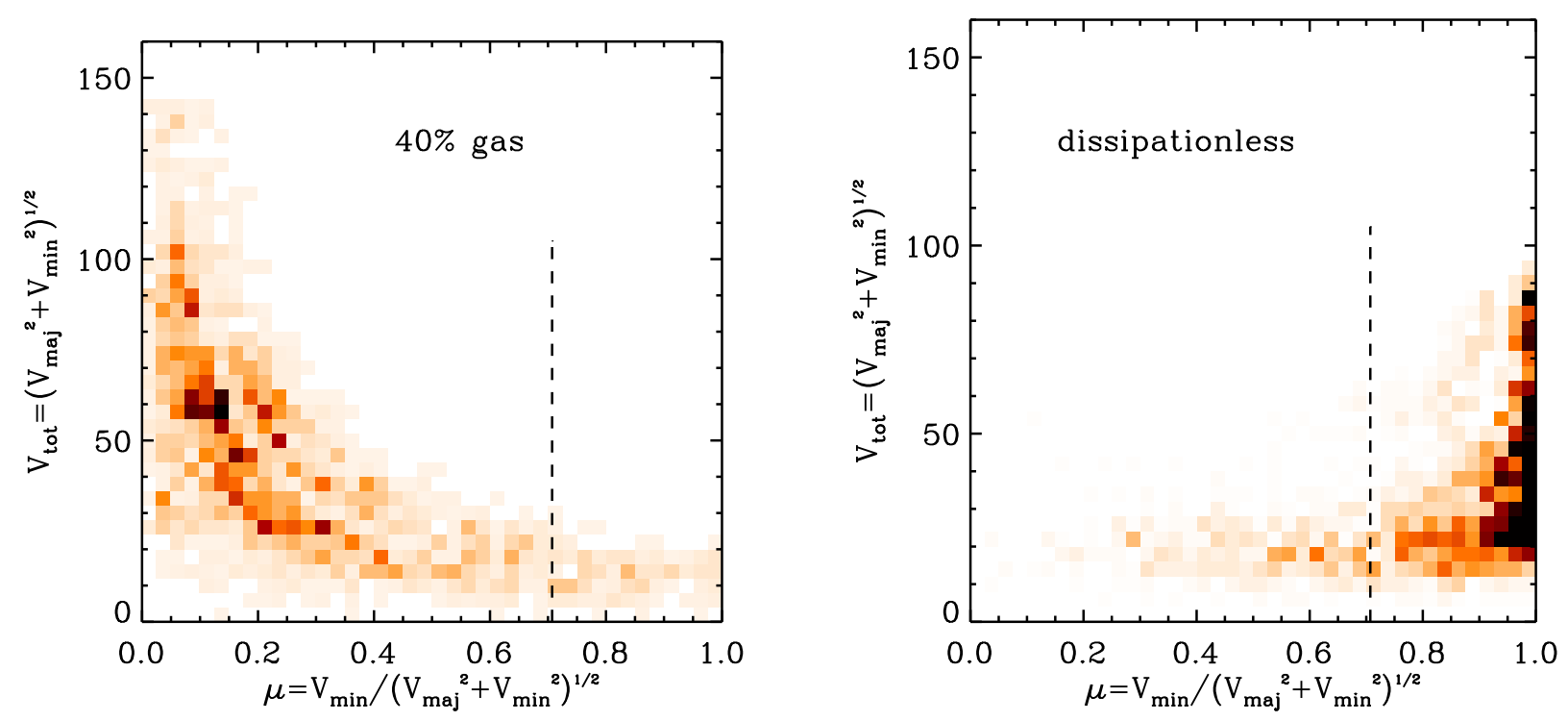

FIG. 12.- The total rotation velocity plotted against the minor axis rotation parameter, $\mu$, as defined by Equation 5 The dashed vertical line denotes equivalent rotation along the major $V_{\text {maj }}$ and minor $V_{\min }$ axes.

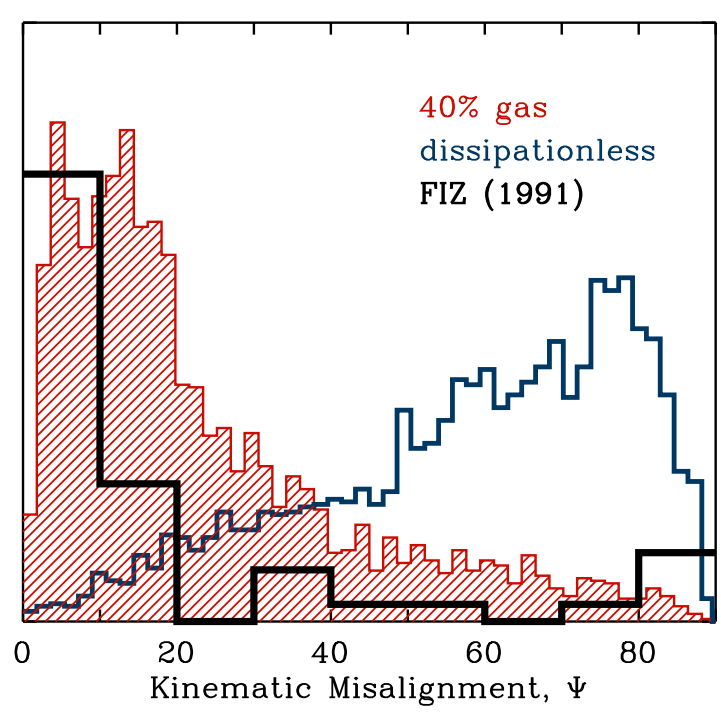

FIG. 13.- Histogram of the kinematic misalignment angle $\Psi$. As with the previous figures, the filled histogram is for dissipational remnants and the open histogram is for dissipationless remnants. Overplotted is data from 44 ellipticals compiled by Franx et al. (1991).

black histogram. While it is clear that there are elliptical galaxies with observed minor axis rotation, the majority (77\%) have $\Psi<20^{\circ}$. There seems to be a large peak of galaxies with little or no observed minor axis rotation, $\Psi \sim 0$, and another, much smaller, peak at $\Psi \sim 90$, although the latter contains only four galaxies, and thus its significance is difficult to assess. In any case, the observed distribution of kinematic misalignments is much better represented by the remnants produced in dissipational mergers.

\subsection{Isophotal Shapes}

The isophotes of elliptical galaxies are not perfect ellipses. The distortions from a perfect ellipse can be quantified by expanding the residuals from the fitted ellipse in a Fourier series,

$$
\Delta r=\sum_{k}\left[a_{k} \cos (k \phi)+b_{k} \sin (k \phi)\right],
$$

where $\Delta r$ are the deviations from a perfect ellipse as a function of angle $\phi$. The coefficient $a_{4}$ of the $\cos (4 \phi)$ term measures deviations symmetric to the major axis. Positive values of $a_{4}$ indicate disky isophotes and negative values indicate boxy isophotes (Bender 1988; Bender et al. 1988).

As mentioned in the introduction, elliptical galaxies appear to come in two types, either disky, or boxy. This dichotomy led Kormendy \& Bender (1996) to suggest a modified Hubble classification system in which ellipticals are delineated by their isophotal shapes as opposed to the standard ellipticity. One line of speculation suggests that disky isophotes are an indication of increased dissipation, and thus the dichotomy in isophotal shapes represents varying amounts of dissipation during a merger (Bender et al. 1992). However, numerical simulations have shown that remnants formed from dissipationless merging can appear both boxy and disky, depending on the viewing angle and isophotal radius (Hevl et al. 1994). It has also been shown that there is a weak trend for dissipational remnants to be more disky (Springel 2000; Bekki \& Shiova 1997) probably owing to the production of central density cusps that destabilize box orbits (Gerhard \& Binnev 1985; Merritt \& Fridman 1996; Barnes \& Hernquist 1996).

We measure the deviations from perfect ellipses for each of our remnant images and list the average for each orientation in Table [5] From these averages we confirm that the dissipational remnants are more disky, in general, than their dissipationless counterparts. We do caution, however, that the scatter within any particular remnant is large, reflecting that remnants can appear disky 
TABLE 5

ISOPHOTAL SHAPES

\begin{tabular}{|c|c|c|}
\hline ID & $\begin{array}{l}\text { Dissipationless } \\
100 a_{4} / a\end{array}$ & $\begin{array}{l}40 \% \text { Gas } \\
100 a_{4} / a\end{array}$ \\
\hline $\mathrm{h}$ & -1.5 & 0.9 \\
\hline b & 0.3 & 1.2 \\
\hline $\mathrm{c}$ & -1.8 & 1.6 \\
\hline $\mathrm{d}$ & -1.3 & 1.8 \\
\hline $\mathrm{e}$ & -1.0 & 0.4 \\
\hline $\mathrm{f}$ & 1.0 & 0.9 \\
\hline $\mathrm{g}$ & 2.5 & 0.9 \\
\hline $\mathrm{i}$ & 0.1 & 1.9 \\
\hline $\mathrm{j}$ & 0.5 & 0.1 \\
\hline $\mathrm{k}$ & -2.5 & 0.8 \\
\hline l & 2.7 & -0.3 \\
\hline $\mathrm{m}$ & 0.4 & -0.8 \\
\hline $\mathrm{n}$ & 0.0 & 1.6 \\
\hline o & -0.9 & 1.7 \\
\hline $\mathrm{p}$ & -0.2 & 1.0 \\
\hline
\end{tabular}

Note. - Average isophotal shape parameter $a_{4} / a$ multiplied by one hundred for each simulated merger remnant. Positive values of $a_{4} / a$ denote disky isophotes, while negative values denote boxy isophotes.

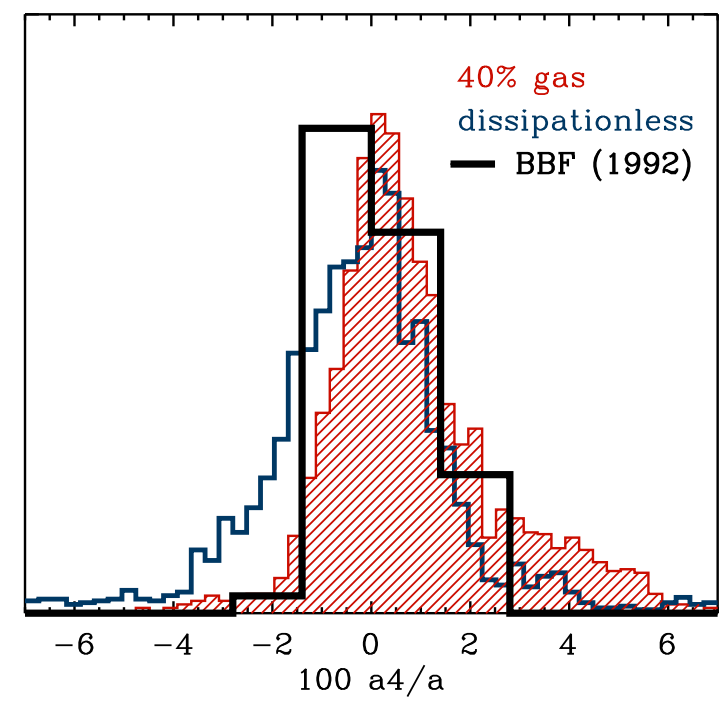

FIG. 14.- Histogram of $100 a_{4} / a$, which measures the isophotal deviations from a perfect ellipse; positive values indicate disky isophotes and negative values indicate boxy isophotes. As with the previous figures the filled histogram is for dissipational merger remnants and the open histogram is for the dissipationless merger remnants. Overplotted is data from 59 ellipticals compiled by Bender et al. (1992).

or boxy depending on the viewing angle.

In Figure 14 we show a histogram of the coefficient $a_{4}$ (normalized by the semi-major axis $a$ and multiplied by an arbitrary factor of one hundred). Also included in this figure is data from 59 ellipticals compiled by Bender et al. (1992). In general, there is good agreement between the simulated merger remnants and the data. While most isophotes having relatively small deviations from perfect ellipses, dissipational remnant tend to be slightly disky and dissipationless remnants slightly boxy. Observationally, there are an equal number of disky and boxy remnants.

We note that there exists a tail of disky dissipational remnants and boxy dissipationless remnants not present in the data. This discrepancy may be a result of resolution, as degrading the resolution of our image, or smoothing it, both reduce the measured isophotal deviation. We also note that many of the disky dissipational remnants are the fast rotating systems viewed edge-on.

As evidence for the dichotomy between disky and boxy ellipticals, Kormendv \& Bender (1996) presented correlations between the $a_{4}$ parameter and $(V / \sigma)^{*}$ and minor axis rotation parameter $\mu$. These correlations are presented in Figure [15] Neither series of remnants presents a clear dichotomy between disky/boxy and rotation, as is seen in the data. The dissipationless remnants, in particular, appear to be an especially poor match to the data as there are a significant number of slow, $(V / \sigma)^{*} \ll 1$, and minor axis, $\mu \sim 1$, rotators with disky isophotes. Elliptical galaxies with these rotational properties are all boxy, in disagreement with the simulations.

Overall, the dissipational remnants are a much better match to the general trends present between the rotation and isophotal shape of elliptical galaxies. Most of these remnants have very little minor axis rotation, $\mu<1$, $(V / \sigma)^{*} \approx 1$, and have isophotes that are both disky and boxy. There are a large number of observational analogs to these systems. However, Figure 15 makes it evident that we are still unable to produce slow and minor axis rotators that are uniformly boxy. This will be discussed further in $\S 4$

\subsection{Dependencies}

The previous sections demonstrate that there is a significant difference between the size, shape and rotational properties of merger remnants when the initial disk contains $40 \%$ gas compared to when the entire disk is dissipationless. However, it is unclear whether these results are sensitive to the various assumptions implicit to our merger simulations. While a complete exploration of the large parameter space that describes the disk galaxy models and their encounters is beyond the scope of this work, we can provide some indications of the robustness of our results by systematically varying several parameters which we suspect may affect the properties of the merger remnants. Specifically, the following three sections consider variations in the progenitor disk gas fraction, the orbital angular momentum, and the size (mass) of the progenitor disk galaxies.

For simplicity we select the $e, h$, and $k$ disk orientations from Table 1 to resimulate throughout this section. These three are selected to span a range of remnant properties.

\subsubsection{Dependence on Gas Fraction}

To begin, we systematically change the disk gas fraction from zero, i.e., the dissipationless case, to a pure gas disk. For reference, our fiducial dissipational simulation contained a disk with $40 \%$ gas. In Figure 16 we show the size, as measured by the semi-major axis, ellipticity, and $(V / \sigma)^{*}$, all averaged over all projections for each merger remnant. In addition, we show the triaxiality parameter $T$, and the anisotropy $\delta$ for each remnant.

As might be expected from the results of $\S 3$ there is a systematic trend for remnants produced by the collision 

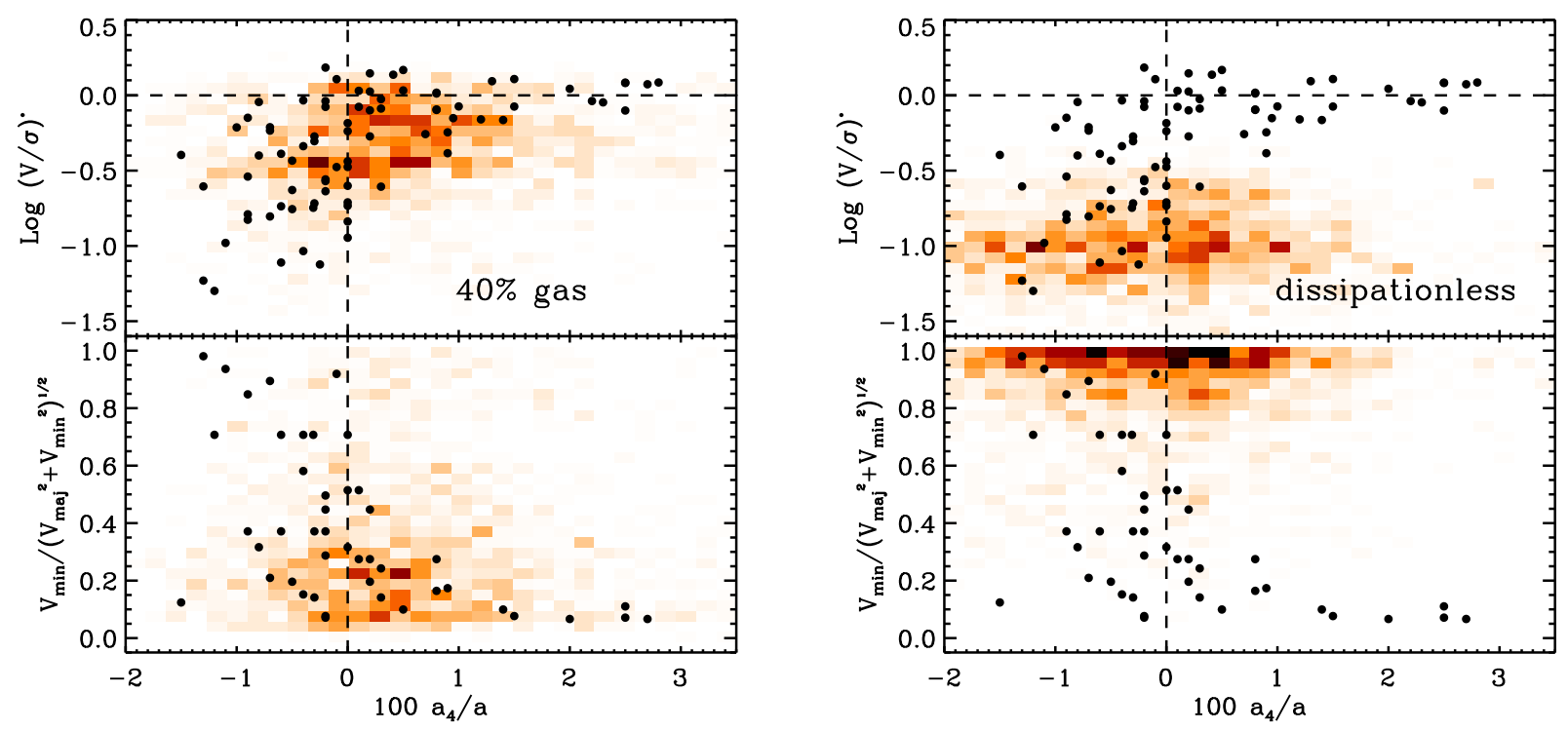

FIG. 15. - A density map that displays correlations between isophotal shape and rotation. $100 a_{4} / a$, which measures the isophotal deviations from a perfect ellipse; positive values indicate disky isophotes and negative values indicate boxy isophotes. The top panel shows the rotation parameter $(V / \sigma)^{*}$ and the lower panel shows the minor axis rotation parameter of Eq. 5 Overplotted is the identical data as presented in Kormendy \& Bender (1996).

of spiral galaxies with an increasing fraction of gas to be smaller, rounder, and to have more rotation. However, some of the remnant properties are more sensitive to the gas fraction than others. For example, the size of remnants shrinks and $(V / \sigma)^{*}$ steadily rises with increasing gas fraction. But, the projected ellipticity becomes only slightly rounder, and at a level that is comparable to the variations expected from projection effects alone (shown by the error bars on each point). For each individual orientation the shape, as measured by the triaxiality parameter $T$, appears to level off above a gas fraction of $20 \%$, even though the individual orientations level off to different shapes.

The dependencies displayed by trends in $(V / \sigma)^{*}, T$, and $\delta$ demonstrate that inherent differences exist purely because of the initial disk orientations. For example, the $e$ merger tends to be much slower rotating, much more triaxial, and contain much less anisotropy, no matter what the gas fraction is, even though the trends with gas fraction are similar to those found in $h$ and $k$ mergers.

From this series of 21 simulations ( 3 orientations, each simulated with 7 different gas fractions) we conclude that the size and rotation are strongly affected by the initial disk gas fraction, while the shape is only moderately affected. The anisotropy appears more strongly correlated with the orbital configuration than with the gas fraction.

These results also directly relate to those of Robertson et al. (2006a) who find that remnants produced in major mergers can reproduce the observed fundamental plane of ellipticals when the progenitor disks contain gas fractions greater than $30 \%$. Thus, it seems that gas-rich major mergers can help explain both the kinematics of elliptical galaxies and the tilt of the fundamental plane.

\subsubsection{Dependence on Orbital Angular Momentum}

Next, we note that merger orbits as determined by cosmological simulations span a range of impact param- eters (Khochfar \& Burkert 2001). In the fiducial series of mergers, analyzed in $\S$ B we assumed each merger orbit was parabolic, and had a pericentric distance of $7.1 \mathrm{kpc}$, generating an orbit with a low impact parameter. In this series of runs, we resimulate the dissipational mergers $(e, h$, and $k)$ with a range of pericenter separations between 3.6 and $57 \mathrm{kpc}$. The initial starting separation for all mergers was $143 \mathrm{kpc}$. We note that all of these orbits contain an identical amount of energy (zero) yet have progressively more orbital angular momentum $\left(L^{2} \sim R_{\text {peri }}\right)$.

Figure 17 is equivalent to Figure [16] except in the present figure the horizontal axis measures the pericentric distance. The size of each merger remnant is a moderate function of $R_{\text {peri }}$, with wide orbits producing larger remnants. The apparent ellipticity has no discernible correlation with $R_{\text {peri }}$. The rotation shows weak, or no, correlation to $R_{\text {peri }}$. At first glance, this lack of correlation between rotation and orbital angular momentum seems odd. However, two effects act to offset the transfer of orbital angular momentum to remnant rotation. First, the initial disk galaxies contain massive dark halos which soak up the majority of the orbital angular momentum. Second, the orbits with high orbital angular momentum require a longer time to merge, and thus the initial disks have consumed a larger fraction of their initial gas. In this sense, they are effectively lower gas fraction mergers, which the previous section showed are slower rotators.

As we saw in the last section, the shapes of merger remnants depend on the original disk orientations. Figure 17 demonstrates that the shape also depends on $R_{\text {peri }}$. Wide orbits tend to produce prolate $(T \approx 1)$ remnants, regardless of the initial disk orientation, while intermediate and radial orbits produce remnants that are closer to oblate. However, the orbits with $R_{\text {peri }}<30 \mathrm{kpc}$ begin to segregate based upon the initial disk orientation. There is a trend, though, for very radial orbits to be more prolate 


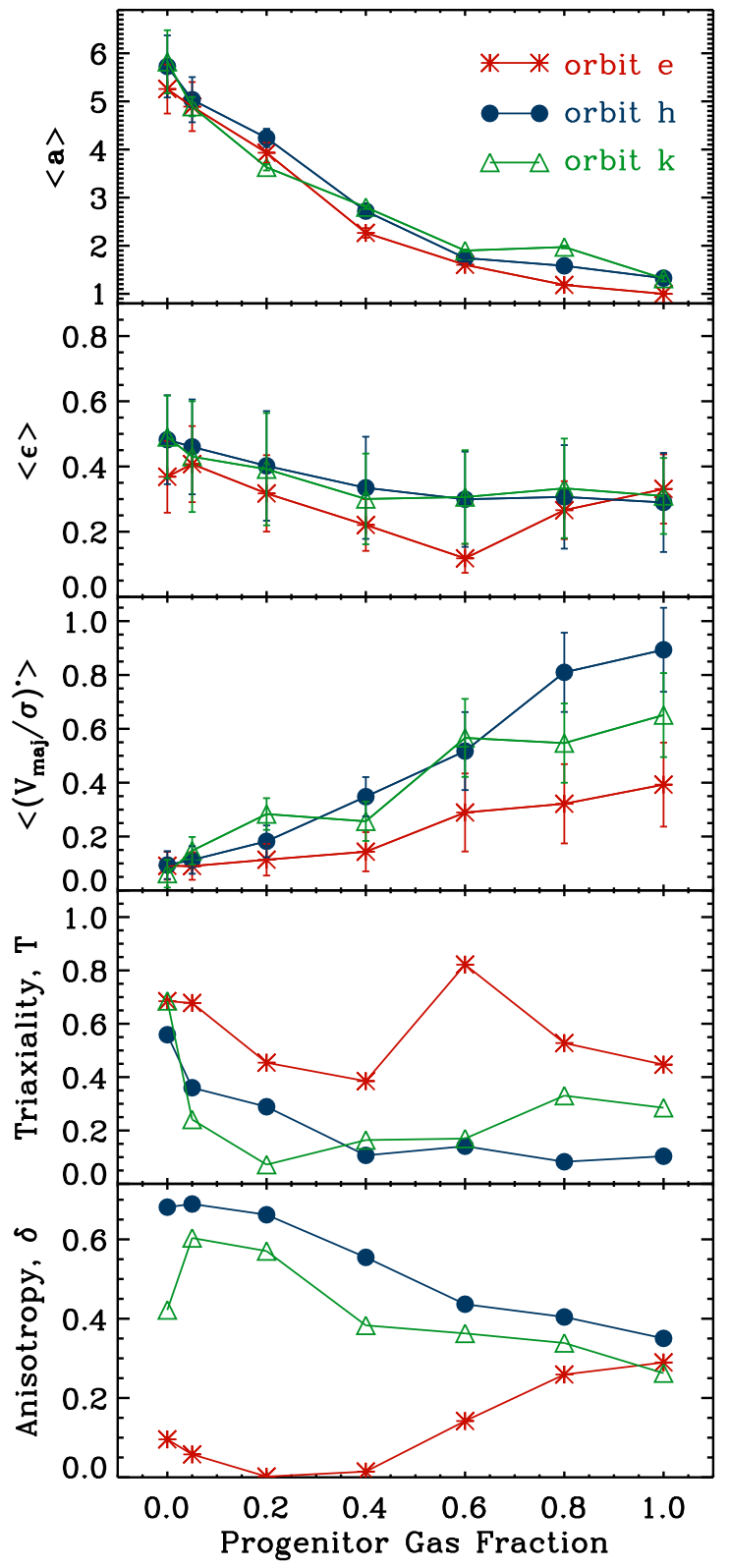

FIG. 16. - The semi-major axis $a$, ellipticity $\epsilon$, and $\mathrm{V}_{\mathrm{maj}} / \sigma$ averaged over 190 different lines of sight and the triaxiality parameter $T$, and anisotropy parameter $\delta$ for our $e, h$ (prograde-prograde), and $k$ orientation merger remnants as a function of the gas fraction of the progenitor disk. A key is provided in the top panel which denotes the symbols used for the three initial disk orientations. For the top three panels, which present quantities averages over many lines of sight, one sigma error bars are included to provide a sense of the variation introduced by projection effects.

than intermediate orbits.

There is a mild tendency for very wide orbits to produce more isotropic $(\delta \sim 0)$ remnants. This correlation, however, is weak, at best, especially compared to the strong dependence on the initial disk orientations.

\subsubsection{Dependence on Progenitor Mass}

As a final dependency we investigate the relation between progenitor mass and the remnant properties. In order to span a wide range of remnant central

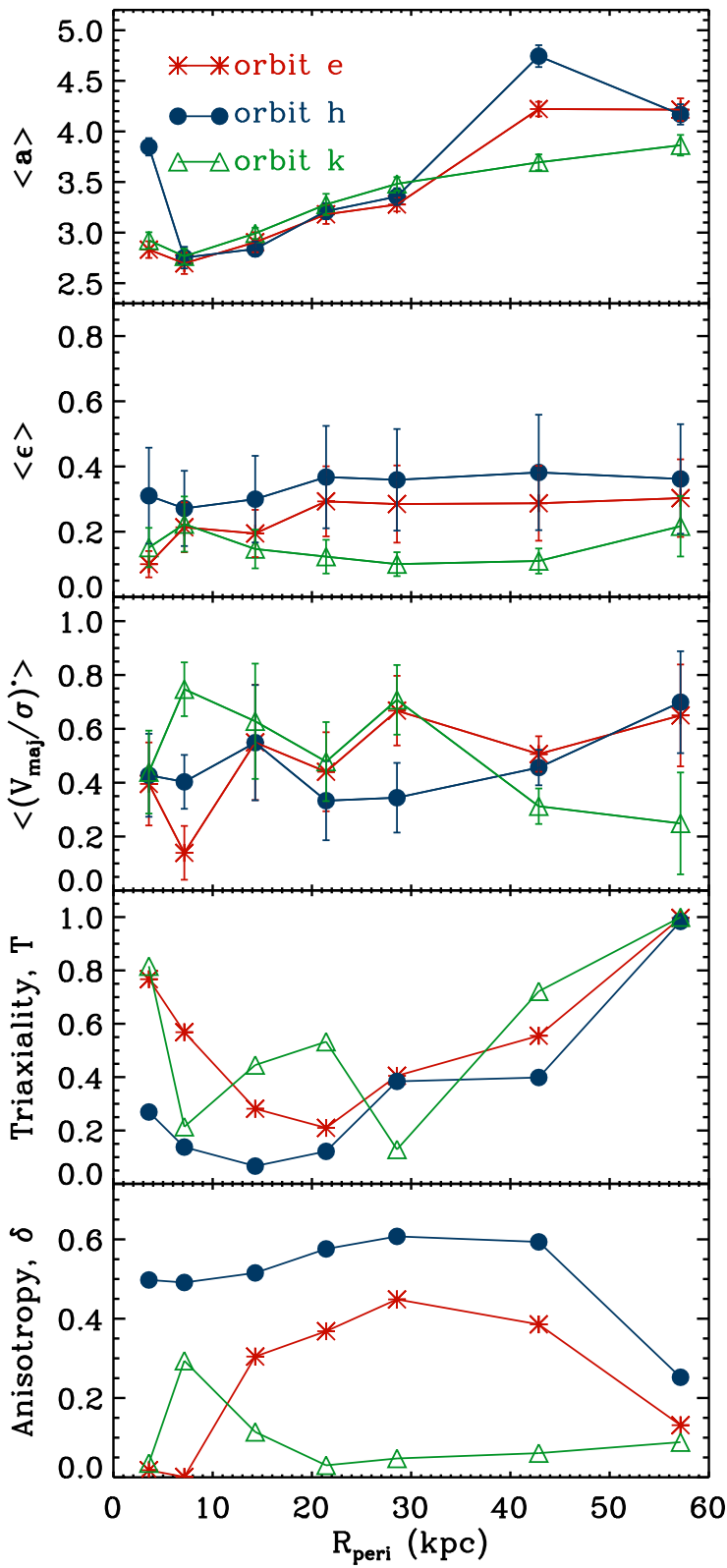

FIG. 17.- Same as Figure 16 except here the remnant properties are plotted against the orbital pericentric distance.

velocity dispersions, we have simulated our standard $e, h$, and $k$ mergers with initial disk galaxies both smaller $\left(V_{200}=56,80,115 \mathrm{~km} \mathrm{~s}^{-1}\right)$ and larger $\left(V_{200}=\right.$ $\left.220,320,500 \mathrm{~km} \mathrm{~s}^{-1}\right)$ than our fiducial case $\left(V_{200}=\right.$ $160 \mathrm{~km} \mathrm{~s}^{-1}$ ). For these mass excursions, all other progenitor disk properties are kept fixed, such as gas fraction (40\%), spin (0.033), dark matter concentration (9), and specific angular momentum (0.05). The initial orbits are scaled such that the ratio $R_{\text {peri }} / R_{d}$ remains constant. For reference, our fiducial model $\left(V_{200}=160 \mathrm{~km} \mathrm{~s}^{-1}\right)$ has a total virial mass of $1.4 \times 10^{12} \mathrm{M}_{\odot}$, and our mass excursion samples galaxies a factor of 20 smaller and a factor of 30 larger in mass.

Figure 18 shows the resulting remnant properties as a function of remnant central velocity dispersion. In gen- 


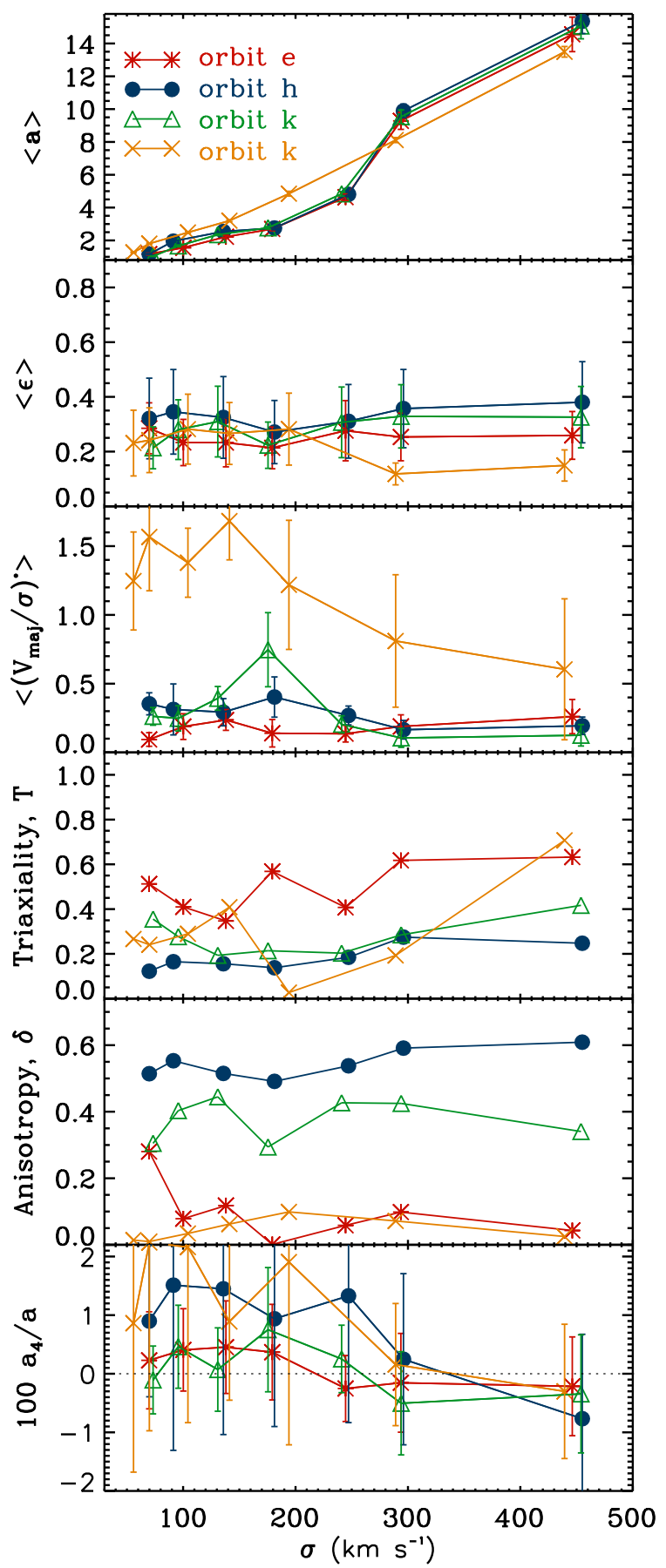

Fig. 18. - Same as Figure 16 except here the remnant properties are plotted against the central velocity dispersion $\sigma$ of each merger remnant.

eral, it appears as if the size is the strongest dependency, as expected since $R_{d} \propto V_{200}$. We find a size-mass relationship consistent with Robertson et al. (2006ba), who simulate binary mergers for an equivalent range of progenitor disk masses. The ellipticity and rotation do not appear to correlate with mass. Once again, the triaxiality and anisotropy depend on the initial disk orientations more than on the size of the merging systems.

From our investigation of the remnant properties as a function of progenitor mass we conclude that mass is of little importance in fixing the rotational properties of a merger remnant. Instead, it is the progenitor gas fraction and merger orbit and orientation which are the primary determinants in what is left behind from the collision of two disk galaxies.

\subsubsection{Other Dependencies}

In the three previous subsections we have tried to determine the degree to which our results are dictated by our initial assumptions. We conclude that the initial gas fraction is of critical importance to the structure of a merger remnant. The orbit and orientation, as well as the progenitor disk mass are also factors which determine the remnant properties. However, there are still a large number of parameters (bulge size, radial gas profile, halo $\lambda$ and $c$ ) which may in principle influence the remnants that have not been addressed so far.

We have performed a single simulation where the initial disk contained a bulge one-third the mass of the progenitor disk. The fiducial disk galaxy model did not contain a bulge. In this case the remnant showed systematic, but very small differences from the no-bulge fiducial remnant. Specifically, because of the dense central bulge, the remnant is rounder and has a higher central velocity dispersion. The no-bulge remnant also has more rotation, but because $\sigma$ increases, the net effect is to lower $V / \sigma$, and drive the remnant down and to the left in the anisotropy diagram. The presence of a bulge increases the rotation because star formation is suppressed during the early stages of the merger Mihos \& Hernquist 1994c, 1996) and hence the effective gas fraction at the final merger is larger than in the no-bulge case. We expect that any process, or assumption, that results in a high gas fraction during the final merger will increase the rotation of the remnant. Possible mechanisms are the inclusion of a bulge, less efficient quiescent star formation, or an extended gas distribution.

We finally note that the presence of a black hole has very little effect to the results presented here. The black hole feedback induced "blowout" phase is necessary to terminate star formation and thereby produce red remnants (Springel et al. 2005a), but this process occurs after the merger has taken place and subsequent to the production of most of the remnant stars. In fact, we ran one case that did not include black hole feedback, and thus did not produce a galactic wind, however the remnant stellar mass increased by less than $5 \%$. While these additional stars add to the "young" component shown in Figure 7 the overall changes to the remnant kinematics are much less than projection effects or merger orientations. Thus we conclude that the black hole may be very important for the long-term color evolution, but has little importance in determining the bulk kinematics of the remnant because the processes that fix the dynamics have finished before the blowout.

\section{DISCUSSION}

The previous section presented an analysis of two series of fifteen merger simulations, one series consisted of dissipationless mergers, while the second series contained disks composed of $40 \%$ gas, but identical in every other respect. In addition, we investigate the dependence of our results to variations in the original gas fraction, orbital angular momentum, and progenitor size/mass. 
These tests show that the size, shape and rotation of any merger remnant is a strong function of the initial disk gas fraction and the orientations of the disks. In what follows we will attempt to place these results into a broader context and relate them to our current understanding of the formation and evolution of spheroidal galaxies.

\subsection{Forming Low-luminosity Elliptical Galaxies and Bulges}

As outlined in the Introduction, low-luminosity ellipticals and bulges fall into the class of galactic spheroids that have disky isophotes, are X-ray faint, have powerlaw surface brightness profiles and show significant rotation. The rotation axis is typically aligned with their photometric minor axis and they are consistent with being oblate isotropic rotators. Thus, asking "What mechanism forms low-luminosity ellipticals and bulges?" may be equivalent to the question "What produces oblate isotropic rotators?"

Our results suggest that the merger of equal mass gasrich disk galaxies may produce remnants that are oblate isotropic rotators. Qualitatively, over $25 \%(4 / 15)$ of the $40 \%$ gas major mergers yielded an oblate isotropic rotator. However, it is unclear if major mergers can produce these objects in the correct number. As indicated by Figure 6] the dissipational mergers produce slightly fewer fast rotating, $(V / \sigma)^{*} \geq 1$, ellipticals than observed. Does this dearth of fast rotating remnants pose a problem to the "merger hypothesis"?

The short answer to this question is no. The scenario outlined in the current paper, major mergers between gas-rich disk galaxies, is likely only one of a number of possible mechanisms to produce oblate isotropic rotators. Another possible mechanism, proposed by Naab \& Burkert (2003), is the dissipationless merger of unequal mass disk galaxies. Both Naab \& Burkert (2003) and Bournaud et al. (2004, 2005) show that unequal mass mergers, specifially those with mass ratios 1:4 or higher, produce oblate remnants.

While we consider there to be strong evidence that all mergers should include progenitor galaxies with some amount of gas, we suspect that the inclusion of gas will only increase the amount of rotation in the remnants of minor mergers. Finally, we note that Figure 16 shows that the frequency of oblate isotropic rotators will increase when initial disks of higher gas fraction are merged. In short, it does not apear that there are any problems in producing oblate isotropic spheroids.

It is also noteworthy that not all of the gas is consumed by the merger event. In general, $\sim 10-20 \%$ of the original gas mass remains in the remnant, typically in a hot phase that is spread throughout the dark halo (Cox et al. 2004, 2006). The cooling time of this gas is several Gyr, and thus, depending on the angular momentum of this gaseous material, and the amount of pristine inter-galactic material that is newly accreted, it is possible a new gas disk will from around, or within, the existing spheroidal stellar remnant. In this sense the remnants produced here may be the precursor to bulges in present day spirals or at least a hidden disk component.

\subsection{Forming Large Elliptical Galaxies}

Luminous elliptical galaxies have boxy isophotes, contain halos of hot gas, have surface-brightness profiles with cores and demonstrate little or no rotation. While forming low-luminosity ellipticals was equivalent to forming oblate isotropic rotators, an analogous equation does not exist for luminous ellipticals. We do note that the primary conclusion of our work is that dissipational merger remnants are a much better match to the entire class of elliptical galaxies. The evidence for this comes from the distribution of ellipticities (Figure 3) and $(V / \sigma)^{*}$ (Figure 6), and the amount of minor axis rotation (Figure 13), as measured by $\Psi$, the kinematic misalignment as well as the correlations between rotation and isophotal shape (Figure 15). However, as the isophotal analysis demonstrates, it is unclear that any of our remnants, dissipational or dissipationless, are really slow rotators and uniformly boxy as it appears all luminous ellipticals are. This difficulty with the model brings into question whether luminous ellipticals can be produced within the merger hypothesis.

While a single gas-rich merger appears to well reproduce the properties of low-luminosity ellipticals, it is possible that luminous ellipticals require a more complicated merger history. In fact, within the hierarchical build-up of structure predicted by Lambda cold dark matter, we expect mergers to occur frequently, especially minor mergers, and there is no reason to believe that galaxies participate in only one major merger. Recent observations suggest that, on average, somewhere between one-half and all elliptical galaxies undergo a spheroid-spheroid merger van Dokkum 2005; Bell et al. 2005) below redshift one. In the context of forming bright cluster galaxies, multiple dry mergers has long been a well-motivated mechanism (Merritt 1985; Dubinski 1998). As a brief test of the effects of continued merging, we performed one re-merger, where two merger remnants were merged in a manner similar to our fiducial series. In this one case, the remnant rotation and ellipticity decreased slightly, while the central velocity dispersion increased slightly and the isophotes became increasingly boxy. Although it is difficult to say anything concrete from a single simulation, we speculate that spheroid-spheroid merging will move remnants vertically downwards in the anisotropy diagram to the region occupied by luminous slowly-rotating ellipticals while also making the system uniformly boxy. A similar result has been found for mergers of dissipationless remnants by Naab et al. (2006) and we note that other work has also shown that multiple merger remnants can remain on the fundamental plane (Capelato et al. 1995; Nipoti et al. 2003; González-García \& van Albada 2003; Bovlan-Kolchin et al. 2006; Robertson et al. 2006a) and may have $R^{1 / 4}$ surface brightness profiles (Bournaud et al. 2005). However, it is clear that much more work needs to be performed in order to understand the relationship between different merger histories and the kinematics of the remnant.

\subsection{Future Considerations}

As a final point of discussion we note that a considerable amount of work still needs to be performed in order to prove that the simulated merger remnants are bona fide elliptical galaxies. The present study has shown that dissipational merger remnants appear to be a good 
match (at least much better than dissipationless remnants) to the ellipticities, $(V / \sigma)^{*}$, and kinematic misalignments $\Psi$ observed in elliptical galaxies. However, we have not addressed the surface brightness profiles, metallicities and abundance ratios, and kinematic subsystems of our merger remnants. Work toward this end is currently underway and is necessary as many of these features are observed to be correlated with the rotational properties suggesting that all of these properties are a result of a common formation mechanism.

\section{CONCLUSIONS}

In this paper we have analyzed a series of numerical simulations to investigate the kinematic structure of galaxies formed from the collision of equal-mass disk galaxies. In particular, we determine that remnants produced by the collision of gas-rich disk galaxies are smaller, rounder, have higher central velocity dispersions (on average) and larger rotation speeds than the remnants of dissipationless disk galaxy mergers.

The larger rotation present in gas-rich merger remnants owes its origin to dissipation. Stars formed during the merger rotate faster than stars present before the merger began. Dissipation and star formation also produce remnants that are closer to oblate and are uniformly more isotropic than dissipationless remnants. Slightly more than one-quarter of the dissipational merger remnants are consistent with being oblate isotropic rotators.

Compared to observed ellipticals, the remnants formed from dissipational mergers are a significantly better match to the distribution of ellipticities, $(V / \sigma)^{*}$, and kinematic misalignment angles $\Psi$ of elliptical galaxies than dissipationless merger remnants. In particular, dissipationless remnants demonstrate significant minor axis rotation and appear to be flattened much more than observed ellipticals.

We also calculate the isophotal shapes of the simulated merger remnants. Dissipational remnants tend to be disky while dissipationless remnants tend to be boxy. Observed ellipticals are evenly distributed between disky and boxy. While both remnants appear to be a sufficient match to the observed distribution, when comparing the correlation between disky/boxy and rotation, the dissipationless merger produce a significant number of slowly rotating disky remnants where there are no observed analogs. Both dissipational and dissipationless mergers produce remnants that are both disky and boxy, and thus these mechanisms have difficulty reproducing the luminous, slowly rotating ellipticals that are observed to be uniformly boxy.

In general, our results suggest that dissipationless disk galaxy mergers cannot be the dominant mechanism to form elliptical galaxies. Dissipational mergers, on the other hand, appear to be a viable mechanism to produce elliptical galaxies, specifically oblate isotropic rotators (i.e., low-luminosity ellipticals), and thus our results lend support to the "merger hypothesis" provided that the progenitor disk galaxies are gas-rich. As mentioned in $\S 11$ additional evidence for the dissipative origin of ellipticals comes from their high phase space density compared to spiral galaxies. Gas dissipation provides a natural mechanism to increase the phase space density during the merger and also appears to be necessary for reproducing the scaling relation of elliptical galaxies (Robertson et al. 2006a).

Our modeling suggests several avenues for further testing this hypothesis. Mergers between gas-rich spirals will imprint subtle features into the remnants. The central starburst will modify the inner profiles of the remnants (Mihos \& Hernquist 1994a), perhaps accounting central light excesses seen in merging systems (Rothberg \& Joseph 2004, 2006). In principle, this can be tested by comparing predictions for metallicity and color gradients with observations (Mihos \& Hernquist 1994b). Dissipational merger may also provide a natural mechanism to produce kinematic subsystems in elliptical galaxies (Hernquist \& Barnes 1991; Bendo \& Barnes 2000). The shells, ripples, loops and other fine structures seen around many relaxed ellipticals (Schweizer 1998) are a natural consequence of mergers involving disk galaxies (Hernquist \& Spergel 1992), but that do not form in major mergers between hot stellar systems. Determining the ubiquity of fine structure in red galaxies would further constrain the importance of disk mergers to the formation of ellipticals.

Placed within the "cosmic cycle" of galaxy formation, we can now argue that gas-rich major mergers trigger quasars and starbursts, fuel the growth of supermassive black holes, and produce remnant galaxies which have the colors, scaling relations, and kinematics akin to present day ellipticals.

We thank Marijn Franx for the motivation that initiated this work and additional comments that significantly improved this paper. We also thank Ralf Bender for providing the data in Figure 15] This work was supported in part by NSF grants ACI 96-19019, AST 0071019, AST 02-06299, and AST 03-07690, and NASA ATP grants NAG5-12140, NAG5-13292, and NAG513381. The simulations were performed at the Center for Parallel Astrophysical Computing at HarvardSmithsonian Center for Astrophysics.

\section{REFERENCES}

Alam, S. M. K. \& Ryden, B. S. 2002, ApJ, 570, 610

Allgood, B., Flores, R. A., Primack, J. R., Kravtsov, A. V., Wechsler, R. H., Faltenbacher, A., \& Bullock, J. S. 2006, MNRAS, 367, 1781

Barnes, J. E. 1988, ApJ, 331, 699

-. 1992, ApJ, 393, 484

Barnes, J. E. \& Hernquist, L. 1996, ApJ, 471, 115

Barnes, J. E. \& Hernquist, L. E. 1991, ApJ, 370, L65

Bekki, K. \& Shioya, Y. 1997, ApJ, 478, L17+
Bell, E. F., Naab, T., McIntosh, D. H., Somerville, R. S., Caldwell, J. A. R., Barden, M., Wolf, C., Rix, H.-W., Beckwith, S. V. W., Borch, A., Haeussler, B., Heymans, C., Jahnke, K., Jogee, S., Meisenheimer, K., Peng, C. Y., Sanchez, S. F., \& Wisotzki, L. 2005, ArXiv Astrophysics e-prints

Bender, R. 1988, A\&A, 193, L7

Bender, R., Burstein, D., \& Faber, S. M. 1992, ApJ, 399, 462

Bender, R., Doebereiner, S., \& Moellenhoff, C. 1988, A\&AS, 74, 385

Bender, R. \& Nieto, J.-L. 1990, A\&A, 239, 97 
Bender, R., Surma, P., Doebereiner, S., Moellenhoff, C., \& Madejsky, R. 1989, A\&A, 217, 35

Bendo, G. J. \& Barnes, J. E. 2000, MNRAS, 316, 315

Binney, J. 1978, MNRAS, 183, 501

-. 1985, MNRAS, 212, 767

Binney, J. \& Tremaine, S. 1987, Galactic dynamics (Princeton, NJ, Princeton University Press, 1987, 747 p.)

Bournaud, F., Combes, F., \& Jog, C. J. 2004, A\&A, 418, L27

Bournaud, F., Jog, C. J., \& Combes, F. 2005, A\&A, 437, 69

Boylan-Kolchin, M., Ma, C.-P., \& Quataert, E. 2005, MNRAS, 362, 184

-. 2006, astro-ph/0601400

Burkert, A. \& Naab, T. 2005, MNRAS, 363, 597

Capelato, H. V., de Carvalho, R. R., \& Carlberg, R. G. 1995, ApJ, 451,525

Cappellari, M., Bacon, R., Bureau, M., Damen, M. C., Davies, R. L., de Zeeuw, P. T., Emsellem, E., Falcón-Barroso, J., Krajnović, D., Kuntschner, H., McDermid, R. M., Peletier, R. F., Sarzi, M., van den Bosch, R. C. E., \& van de Ven, G. 2006, MNRAS, 366, 1126

Carlberg, R. G. 1986, ApJ, 310, 593

Cox, T. J., Di Matteo, T., Hernquist, L., Hopkins, P. F., Robertson, B., \& Springel, V. 2006, ApJ accepted (astro-ph/0504156)

Cox, T. J., Jonsson, P., Primack, J., \& Somerville, R. S. 2005, MNRAS, submitted (astro-ph/0503201)

Cox, T. J., Primack, J., Jonsson, P., \& Somerville, R. S. 2004, ApJ, 607, L87

Cretton, N., Naab, T., Rix, H., \& Burkert, A. 2001, ApJ, 554, 291

Davies, R. L. \& Birkinshaw, M. 1988, ApJS, 68, 409

Davies, R. L., Efstathiou, G., Fall, S. M., Illingworth, G., \& Schechter, P. L. 1983, ApJ, 266, 41

de Vaucouleurs, G. 1948, Annales d'Astrophysique, 11, 247

de Zeeuw, P. T., Bureau, M., Emsellem, E., Bacon, R., Marcella Carollo, C., Copin, Y., Davies, R. L., Kuntschner, H., Miller, B. W., Monnet, G., Peletier, R. F., \& Verolme, E. K. 2002, MNRAS, 329, 513

de Zeeuw, T. \& Franx, M. 1991, ARA\&A, 29, 239

Di Matteo, T., Springel, V., \& Hernquist, L. 2005, Nature, 433, 604

Dubinski, J. 1998, ApJ, 502, 141

Erb, D. K., Steidel, C. C., Shapley, A. E., Pettini, M., Reddy, N. A., \& Adelberger, K. L. 2006, ApJ accepted (astro-ph/0604041)

Faber, S. M., Tremaine, S., Ajhar, E. A., Byun, Y., Dressler, A., Gebhardt, K., Grillmair, C., Kormendy, J., Lauer, T. R., \& Richstone, D. 1997, AJ, 114, 1771

Faber, S. M., Wegner, G., Burstein, D., Davies, R. L., Dressler, A., Lynden-Bell, D., \& Terlevich, R. J. 1989, ApJS, 69, 763

Ferrarese, L. \& Merritt, D. 2000, ApJ, 539, L9

Franx, M., Illingworth, G., \& de Zeeuw, T. 1991, ApJ, 383, 112

Franx, M., Illingworth, G., \& Heckman, T. 1989, ApJ, 344, 613

Gebhardt, K., Bender, R., Bower, G., Dressler, A., Faber, S. M., Filippenko, A. V., Green, R., Grillmair, C., Ho, L. C., Kormendy, J., Lauer, T. R., Magorrian, J., Pinkney, J., Richstone, D., \& Tremaine, S. 2000, ApJ, 539, L13

Gerhard, O. E. \& Binney, J. 1985, MNRAS, 216, 467

González-García, A. C. \& Balcells, M. 2005, MNRAS, 357, 753

González-García, A. C. \& van Albada, T. S. 2003, MNRAS, 342, L36

-. 2005, MNRAS, 361, 1043

Górski, K. M., Hivon, E., Banday, A. J., Wandelt, B. D., Hansen, F. K., Reinecke, M., \& Bartelmann, M. 2005, ApJ, 622, 759

Hernquist, L. 1990, ApJ, 356, 359

-. 1992, ApJ, 400, 460

-. 1993, ApJ, 409, 548

Hernquist, L. \& Barnes, J. E. 1991, Nature, 354, 210

Hernquist, L. \& Spergel, D. N. 1992, ApJ, 399, L117

Hernquist, L., Spergel, D. N., \& Heyl, J. S. 1993, ApJ, 416, 415

Heyl, J. S., Hernquist, L., \& Spergel, D. N. 1994, ApJ, 427, 165

Hopkins, P. F., Hernquist, L., Cox, T. J., Di Matteo, T., Martini, P., Robertson, B., \& Springel, V. 2005a, ApJ, 630, 705

Hopkins, P. F., Hernquist, L., Cox, T. J., Di Matteo, T., Robertson, B., \& Springel, V. 2005b, ApJ, 630, 716
-. 2005c, ApJ, 632, 81

- 2006a, ApJS, 163, 1

Hopkins, P. F., Hernquist, L., Cox, T. J., Robertson, B., Di Matteo, T., \& Springel, V. 2006b, ApJ, 639, 700

Hopkins, P. F., Hernquist, L., Cox, T. J., Robertson, B., \& Springel, V. 2006c, ApJS, 163, 50

Hopkins, P. F., Hernquist, L., Martini, P., Cox, T. J., Robertson, B., Di Matteo, T., \& Springel, V. 2005d, ApJ, 625, L71

Kazantzidis, S., Mayer, L., Colpi, M., Madau, P., Debattista, V. P., Wadsley, J., Stadel, J., Quinn, T., \& Moore, B. 2005, ApJ, 623, L67

Kennicutt, R. C. 1998, ApJ, 498, 541

Khochfar, S. \& Burkert, A. 2001, ApJ, 561, 517

Kormendy, J. \& Bender, R. 1996, ApJ, 464, L119+

Lake, G. 1989, AJ, 97, 1312

Lambas, D. G., Maddox, S. J., \& Loveday, J. 1992, MNRAS, 258, 404

Lauer, T. R., Faber, S. M., Gebhardt, K., Richstone, D., Tremaine, S., Ajhar, E. A., Aller, M. C., Bender, R., Dressler, A., Filippenko, A. V., Green, R., Grillmair, C. J., Ho, L. C., Kormendy, J., Magorrian, J., Pinkney, J., \& Siopis, C. 2005, AJ, 129, 2138

Lidz, A., Hopkins, P. F., Cox, T. J., Hernquist, L., \& Robertson, B. 2006, ApJ, 641, 41

Merritt, D. 1985, ApJ, 289, 18

Merritt, D. \& Fridman, T. 1996, ApJ, 460, 136

Merritt, D. \& Tremblay, B. 1996, AJ, 111, 2243

Mihos, J. C. \& Hernquist, L. 1994a, ApJL, 437, L47

-. 1994b, ApJ, 427, 112

-. 1994c, ApJ, 431, L9

-. 1996, ApJ, 464, 641

Milosavljević, M. \& Merritt, D. 2001, ApJ, 563, 34

Naab, T. \& Burkert, A. 2003, ApJ, 597, 893

Naab, T., Khochfar, S., \& Burkert, A. 2006, ApJ, 636, L81

Negroponte, J. \& White, S. D. M. 1983, MNRAS, 205, 1009

Nipoti, C., Londrillo, P., \& Ciotti, L. 2003, MNRAS, 342, 501

Ostriker, J. P. 1980, Comments on Astrophysics, 8, 177

Rix, H., Carollo, C. M., \& Freeman, K. 1999, ApJ, 513, L25

Robertson, B., Cox, T. J., Hernquist, L., Franx, M., Hopkins, P. F. Martini, P., \& Springel, V. 2006a, ApJ, 641, 21

Robertson, B., Hernquist, L., Bullock, J. S., Cox, T. J., Di Matteo, T., Springel, V., \& Yoshida, N. 2005, arXiv:astro-ph/0503369

Robertson, B., Hernquist, L., Cox, T. J., Di Matteo, T., Hopkins,

P. F., Martini, P., \& Springel, V. 2006b, ApJ, 641, 90

Rothberg, B. \& Joseph, R. D. 2004, AJ, 128, 2098

-. 2006, AJ, 131, 185

Ryden, B. 1992, ApJ, 396, 445

Schmidt, M. 1959, ApJ, 129, 243

Schweizer, F. 1998, in Saas-Fee Advanced Course 26: Galaxies: Interactions and Induced Star Formation, ed. D. Friedli, L. Martinet, \& D. Pfenniger

Sersic, J. L. 1968, Atlas de galaxias australes (Cordoba, Argentina: Observatorio Astronomico, 1968)

Shen, S., Mo, H. J., White, S. D. M., Blanton, M. R., Kauffmann, G., Voges, W., Brinkmann, J., \& Csabai, I. 2003, MNRAS, 343, 978

Springel, V. 2000, MNRAS, 312, 859

-. 2005, MNRAS, 364, 1105

Springel, V., Di Matteo, T., \& Hernquist, L. 2005a, ApJ, 620, L79

- 2005b, MNRAS, 361, 776

Springel, V. \& Hernquist, L. 2002, MNRAS, 333, 649

-. 2003, MNRAS, 339, 289

Toomre, A. 1977, in Evolution of Galaxies and Stellar Populations, p.401

Toomre, A. \& Toomre, J. 1972, ApJ, 178, 623

Tremblay, B. \& Merritt, D. 1995, AJ, 110, 1039

Trujillo, I., Aguerri, J. A. L., Cepa, J., \& Gutiérrez, C. M. 2001, MNRAS, 321, 269

van Dokkum, P. G. 2005, AJ, 130, 2647 